Prepared For SUBMission to JCAP

\title{
Cosmology with massive neutrinos I: towards a realistic modeling of the relation between matter, haloes and galaxies
}

Francisco Villaescusa-Navarro, ${ }^{a}$ Federico Marulli, ${ }^{b, c, d}$ Matteo Viel, ${ }^{a, e}$ Enzo Branchini, ${ }^{f, g, h}$ Emanuele Castorina, ${ }^{i}$ Emiliano Sefusatti, ${ }^{j, k}$ Shun Saito ${ }^{h}$

${ }^{a}$ INAF - Osservatorio Astronomico di Trieste, Via Tiepolo 11, 34143, Trieste, Italy

${ }^{b}$ Dipartimento di Fisica e Astronomia - Università di Bologna, viale Berti Pichat 6/2, I-40127 Bologna, Italy

${ }^{c}$ INAF - Osservatorio Astronomico di Bologna, via Ranzani 1, I-40127 Bologna, Italy

${ }^{d}$ INFN - Sezione di Bologna, viale Berti Pichat 6/2, I-40127 Bologna, Italy

${ }^{e}$ INFN sez. Trieste, Via Valerio 2, 34127 Trieste, Italy

${ }^{f}$ Dipartimento di Matematica e Fisica, Università degli Studi Roma Tre, via della Vasca Navale 84, 00146 Roma, Italy

${ }^{g}$ INFN - Sezione di Roma Tre, via della Vasca Navale 84, I-00146 Roma, Italy

${ }^{h}$ INAF - Osservatorio Astronomico di Roma, via Frascati 33, I-00040 Monte Porzio Catone (RM), Italy

${ }^{i}$ SISSA - International School For Advanced Studies, Via Bonomea, 26534136 Trieste, Italy

${ }^{j}$ The Abdus Salam International Center for Theoretical Physics, Strada Costiera 11, 34151, Trieste, Italy

${ }^{k}$ INAF, Osservatorio Astronomico di Brera, Via Bianchi 46, I-23807 Merate (LC) Italy

${ }^{h}$ Kavli Institute for the Physics and Mathematics of the Universe (WPI), Todai Institutes for Advanced Study, The University of Tokyo, Chiba 277-8582, Japan 
E-mail: villaescusa@oats.inaf.it

Abstract. By using a suite of large box-size N-body simulations that incorporate massive neutrinos as an extra set of particles, with total masses of $0.15,0.30$, and $0.60 \mathrm{eV}$, we investigate the impact of neutrino masses on the spatial distribution of dark matter haloes and on the distribution of galaxies within the haloes. We compute the bias between the spatial distribution of dark matter haloes and the overall matter and cold dark matter distributions using statistical tools such as the power spectrum and the two-point correlation function. Overall we find a scale-dependent bias on large scales for the cosmologies with massive neutrinos. In particular, we find that the bias decreases with the scale, being this effect more important for higher neutrino masses and at high redshift. However, our results indicate that the scale-dependence in the bias is reduced if the latter is computed with respect to the cold dark matter distribution only. We find that the value of the bias on large scales is reasonably well reproduced by the Tinker fitting formula once the linear cold dark matter power spectrum is used, instead of the total matter power spectrum. We also investigate whether scale-dependent bias really comes from purely neutrino's effect or from nonlinear gravitational collapse of haloes. For this purpose, we address the $\Omega_{\nu}-\sigma_{8}$ degeneracy and find that such degeneracy is not perfect, implying that neutrinos imprint a slight scale dependence on the large-scale bias. Finally, by using a simple halo occupation distribution (HOD) model, we investigate the impact of massive neutrinos on the distribution of galaxies within dark matter haloes. We use the main galaxy sample in the Sloan Digital Sky Survey (SDSS) II Data Release 7 to investigate if the small-scale galaxy clustering alone can be used to discriminate among different cosmological models with different neutrino masses. Our results suggest that different choices of the HOD parameters can reproduce the observational measurements relatively well, and we quantify the difference between the values of the HOD parameters between massless and massive neutrino cosmologies. 


\section{Contents}

1 Introduction 1

2 The simulations $\quad 3$

3 Clustering of dark matter haloes 5

3.1 Halo bias: power spectrum 5

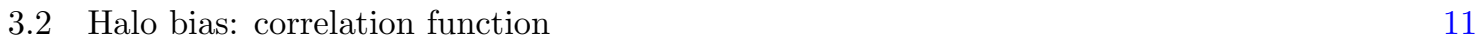

4 Clustering of galaxies $\quad 17$

$\begin{array}{llr}\text { 4.1 Construction of mock galaxy catalogues } & 18\end{array}$

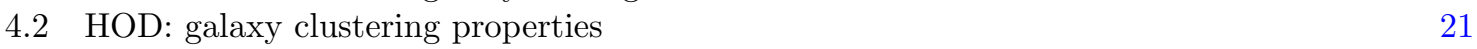

5 Summary and conclusions $\quad 25$

$\begin{array}{ll}\text { A Power spectrum corrections and errors } & 27\end{array}$

B Impact of the halo identification criteria on the halo-matter bias 28

\section{Introduction}

Neutrinos are one of the most interesting and enigmatic particles of the particle standard model. Postulated by Wolfgang Pauli in 1930 to avoid the violation of energy, momentum and spin in the beta decay process, neutrinos were eventually detected in 1956 by Cowan and Reines [1]. Neutrinos have long been considered massless particles until the "oscillation" phenomenon, i.e. the change of flavor, was detected in the neutrinos produced within the sun [2]. The latest results that combine solar, atmospheric and reactor neutrinos allow to constrain the (squared) mass difference between the three neutrino mass eigenstates: $\triangle m_{12}^{2}=7.5 \times 10^{-5} \mathrm{eV}^{2}$ and $\left|\triangle m_{23}^{2}\right|=2.3 \times 10^{-3} \mathrm{eV}^{2}[3,4]$. From the theoretical side, it is of great interest to determine the absolute neutrino mass scale, since this may reveal physics beyond the particle standard model.

From a cosmological point of view it is mandatory to account for the neutrino masses in a precision cosmology era as the one we have just entered. The Big Bang theory predicts the existence of a cosmic neutrino background, and those neutrinos play a fundamental role in setting the primordial abundance of light elements. Moreover, their masses impact on cosmology, at the linear level, on two different ways: modifying the matter-radiation equality and slowing down the growth of matter perturbations. The combination of these effects results in a suppression of the matter power spectra on small scales [5]. The shape of the matter power spectra, constrained on very large scales by the cosmic microwave background and by the galaxy distribution on intermediate scales, has been used to put upper limits on the neutrino masses. Present limits, obtained independently from different galaxy surveys such as $\mathrm{SDSS}^{1}$ and $\mathrm{CFHTLS}^{2}$ point out that $\Sigma_{i} m_{\nu_{i}}<0.3 \mathrm{eV}$ at $2 \sigma[6-15]$, although by using data from the WiggleZ ${ }^{3}$ survey it is found that $\Sigma_{i} m_{\nu_{i}}<0.15 \mathrm{eV}(95 \%)$ [16], and it is expected that those limits shrink to $\Sigma_{i} m_{\nu_{i}} \lesssim 0.03 \mathrm{eV}$, i.e. allowing a determination of the neutrino masses with a significance of $2 \sigma$ even in the normal hierarchy, with future galaxy surveys as Euclid [17-19]. It is worth to note that the latest results from Planck [20] gives an upper limit on the sum of the neutrino masses equal to $\Sigma_{i} m_{\nu_{i}}<0.93 \mathrm{eV}$ within a $95 \%{ }^{4}$ confident interval, assuming a flat $\Lambda$ CDM cosmological model.

Although the impact of neutrino masses on cosmology is very well understood at the linear order $[5,21]$, their impact on the fully non-linear regime has not been extensively studied. The non-linear

\footnotetext{
${ }^{1}$ http://www.sdss.org/

${ }^{2}$ http://www.cfht.hawaii.edu/Science/CFHLS/

${ }^{3}$ http://wigglez.swin.edu.au/site/

${ }^{4}$ This is found with Planck alone, i.e. without combining results with BAO, high-l...etc.
} 
regime is important at both low redshift and small scales, and N-body techniques emerged as the best tools to follow structure formation in it. However, whereas the cold dark matter (CDM), baryons, stars and black holes are commonly simulated in N-body simulations, cosmological neutrinos have not received the same attention.

Recent works have investigated the impact of massive neutrinos on the non-linear regime by using either semi-analytic models or N-body simulations. In particular, those works considered the clustering of relic neutrinos within the gravitational potential wells of CDM haloes [22-26] and the impact of neutrino masses on: the non-linear matter power spectrum [27-35], the Lyman- $\alpha$ forest $[32,36]$, the halo mass function $[24,36,37]$ and the redshift-space distortions [37]. Whereas most of those works are focused on the impact of neutrino masses on large scales, in this paper we explore the effects of massive neutrinos on scales comparable with those of galaxies and dark matter haloes. To achieve that, we have run a large set of N-body simulations containing CDM and neutrinos particles, simulating many different cosmological models characterized by different sums of neutrino masses. Our simulations explore different total neutrino masses (assuming 3 degenerate neutrino families): $0.00,0.15,0.30$ and $0.60 \mathrm{eV}$. Although observational constraints prefer a value of $\Sigma_{i} m_{\nu_{i}}$ smaller than $0.30 \mathrm{eV}$, a value of $0.60 \mathrm{eV}$ is not completely unreasonable, as the latest results of Planck indicate. Moreover, our aim in this paper is not limited to simulate realistic cosmological models, but, more in general, to investigate how massive neutrinos affect the spatial distribution of dark matter haloes and the clustering properties of the galaxies residing in those.

The clustering properties of dark matter haloes in cosmologies with massive neutrinos have already been studied in [37]. In that paper, authors used N-body simulations incorporating neutrinos through the so-called grid method (see [29, 32]). In this method neutrinos only contribute to the long distance force through the particle mesh (PM) method, providing a fast implementation of the matter evolution in neutrino cosmologies, but with the limitation that it does not properly capture the non-linear neutrino regime. The use of the grid method is, thus, only justified on regimes where the non-linear neutrino effects are negligible (at $z \gtrsim 2$ and on large linear scales $[26,29,32]$ ).

In this paper we study the impact of neutrino masses on the halo-matter and galaxy-matter bias by using N-body simulations that incorporates neutrinos as particles (using the so-called particle method). In comparison with the grid-based neutrino N-body simulations, these simulations are better suited to follow the fully non-linear regime. We investigate the halo-matter bias using statistical tools such as the power spectrum and the two-point correlation function. We then populate with realistic galaxies the dark matter haloes of the different cosmological models using a simple halo occupation distribution (HOD) model and investigate the galaxy clustering properties in universes with massive neutrinos. We notice that baryonic effects can also impact on the clustering properties of dark matter haloes and galaxies (see for instance [38]).

This paper is the first of a series of three papers [39, 40]. In Paper II [39] we investigate the universality of the halo mass function (HMF) and of halo bias in cosmologies with massive neutrinos. We show that the abundance of Friends-of-Friends haloes in a massive neutrino model is well reproduced by the Crocce et al. (2010) [41] fitting formula once the CDM linear power spectrum is used to compute $\sigma(M, z)$, rather than the total matter power spectrum. If the above prescription is adopted, then the HMF becomes universal with respect to neutrino masses. Paper II also presents similar results for the large-scale halo bias in presence of massive neutrinos. In particular we show that universality of linear bias factors is recovered only if they are computed with respect to the spatial distribution of the CDM component alone.

In Paper III [17] we verify the findings of Paper II by showing that the HMF of Spherical Overdensity haloes is well described by the Tinker et al. (2008) [42] once the CDM linear matter power spectrum is used to calculate $\sigma(M, z)$. We investigate the effect that the new prescription for the HMF has on cosmological parameters inferred from cluster abundance data, using as a case study the Planck SZ-selected sample of clusters. We find that, for a cosmology with massive neutrinos, the improved HMF calibration provides a stronger degeneracy between the cosmological parameters $\sigma_{8}$ and $\Omega_{m}$, which leads to a lower $\sigma_{8}$ mean value. Taking into account such an effect has the consequences of increasing the tension between the cosmological parameters derived from Planck CMB data and those from cluster number counts [43]. 
The paper is organized as follows. We first describe in section 2 the N-body simulations we have carried out for this work. In section 3 we investigate the impact of neutrino masses on the spatial distribution of dark matter haloes by means of the power spectrum and the correlation function. Then, in section 4, we populate the dark matter haloes of the different cosmological models with galaxies, studying how the galaxy clustering properties are affected by the presence of massive neutrinos. Finally, we present the main conclusions of this work in section 5.

\section{The simulations}

We have run N-body simulations containing CDM and neutrino particles, using the particle-based method, to study the effects of massive neutrinos on the spatial distribution of dark matter haloes and galaxies. As discussed in the above section, the simulations run with this method are better suited for this work, since they allow us to capture the fully non-linear regime, as opposed to the simulations used in [37]. On the other hand, the disadvantage is that the computational cost of running the simulations with this method is much higher than with the grid-based method.

The simulations have been run using the TreePM code GADGET-3, an improved version of the code GADGET-2 [44], using the particle-based implementation. In this implementation, neutrinos are treated as an extra set of particles, in the same way as the CDM, with the difference that, at the starting redshift of the simulation, the neutrinos receive an extra thermal velocity component obtained by random sampling the neutrino Fermi-Dirac linear ${ }^{5}$ momentum distribution. On small scales, the force affecting the neutrinos is computed using the short-range tree. As pointed out in [26], this feature is required to correctly account for the clustering of neutrinos within dark matter haloes and to reproduce the neutrino haloes down to small scales. For further details about this method we refer the reader to $[27,32,34]$. The code time-step is set by the CDM particles, independently on the presence of neutrino particles. The typical total CPU time consumption of our N-body simulations is between 1,000 and 10,000 hours.

The initial conditions of the N-body simulations have been generated at $z=99$, using the Zel'dovich approximation for both the CDM and the neutrino particles. The transfer functions have been obtained through CAMB [45]. We have incorporated the baryon effects (for instance the BAO peaks) into the CDM particles by using a transfer function that is a weighted average of the transfer functions of the CDM and the baryons, the formers as given by CAMB. The gravitational softening of both particle types have been set to $1 / 30$ of their mean inter-particle linear spacing.

In table 1 we list the different simulations we have run for this paper along with the values of their cosmological parameters. The size of our simulation boxes are either 500 or $1000 h^{-1} \mathrm{Mpc}$, chosen to have large statistics but keeping moderate the computational cost. We have used the simulations with large box sizes $\left(1000 h^{-1} \mathrm{Mpc}\right)$ to investigate the spatial distribution of dark matter haloes on large scales, whereas we have used the simulations with $500 h^{-1} \mathrm{Mpc}$ to explore the galaxy clustering properties. The number of CDM particles in the simulations is $512^{3}$, except for a few cases that we have run to perform convergence tests. The masses of the CDM particles for the simulation with the highest resolution, L0-HR, are of $2.07 \times 10^{10} h^{-1} \mathrm{M}_{\odot}$. We have simulated massive neutrino cosmologies with a sum of the neutrino masses equal to $0.15,0.30$ and $0.60 \mathrm{eV}$, assuming that the three neutrino masses are degenerate, to investigate the dependence of our results on the neutrino masses. In simulations containing neutrino particles, the number of those is $512^{3}$, except for the H6-LR series that were run to study the errors when computing the different power spectra and bias. In [26] it was shown that this number of neutrino particles is enough to properly capture the neutrino effects on the large scale structure. For all the simulations we have saved snapshots at $z=0,0.5,1$ and 2.

All the simulations corresponds to flat, $\Omega_{\Lambda}=1-\Omega_{\mathrm{m}}$, cosmological models. Our simulations can be organized into 4 different groups as shown in the table 1 . The simulations belonging to the group 1 (H0-Planck-LV, L6-Planck, L3-Planck, L15-Planck and L0-Planck) share the values of the cosmological parameters $\Omega_{\mathrm{b}}, \Omega_{\Lambda}, h$ and $n_{s}$. Moreover, the value of the parameters $\Omega_{\mathrm{m}}=\Omega_{\mathrm{cdm}}+\Omega_{\mathrm{b}}+\Omega_{\nu}$ and $A_{s}$ (the amplitude of the primordial power spectrum) are the same for all of them. The differences among

\footnotetext{
${ }^{5}$ In [26] it was shown that this distribution is impacted by non-linear effects at low redshift.
} 


\begin{tabular}{|c|c|c|c|c|c|c|c|c|c|c|c|}
\hline Name & $\begin{array}{c}\Sigma_{i} m_{\nu_{i}} \\
(\mathrm{eV})\end{array}$ & $\begin{array}{c}\text { Box } \\
\left(h^{-1} \mathrm{Mpc}\right)\end{array}$ & $\Omega_{\mathrm{m}}$ & $\Omega_{\mathrm{b}}$ & $\Omega_{\nu}$ & $\Omega_{\Lambda}$ & $h$ & $n_{s}$ & $N_{\mathrm{CDM}}^{1 / 3}$ & $N_{\nu}^{1 / 3}$ & $\begin{array}{c}\sigma_{8} \\
(\mathrm{z}=0)\end{array}$ \\
\hline \hline H0-Planck-LV (1) & 0.00 & 1000 & 0.3175 & 0.049 & 0.0 & 0.6825 & 0.6711 & 0.9624 & 1024 & 0 & 0.834 \\
\hline L6-Planck (1) & 0.60 & 500 & 0.3175 & 0.049 & 0.0143 & 0.6825 & 0.6711 & 0.9624 & 512 & 512 & 0.690 \\
\hline L3-Planck (1) & 0.30 & 500 & 0.3175 & 0.049 & 0.0072 & 0.6825 & 0.6711 & 0.9624 & 512 & 512 & 0.761 \\
\hline L15-Planck (1) & 0.15 & 500 & 0.3175 & 0.049 & 0.0036 & 0.6825 & 0.6711 & 0.9624 & 512 & 512 & 0.799 \\
\hline L0-Planck (1) & 0.00 & 500 & 0.3175 & 0.049 & 0.0 & 0.6825 & 0.6711 & 0.9624 & 512 & 0 & 0.834 \\
\hline \hline H6 (8) & 0.60 & 1000 & 0.2708 & 0.050 & 0.0131 & 0.7292 & 0.7 & 1.0 & 512 & 512 & 0.675 \\
\hline H3 (8) & 0.30 & 1000 & 0.2708 & 0.050 & 0.0066 & 0.7292 & 0.7 & 1.0 & 512 & 512 & 0.752 \\
\hline H0 (8) & 0.00 & 1000 & 0.2708 & 0.050 & 0.0 & 0.7292 & 0.7 & 1.0 & 512 & 0 & 0.832 \\
\hline H0-HR (1) & 0.00 & 1000 & 0.2708 & 0.050 & 0.0 & 0.7292 & 0.7 & 1.0 & 768 & 0 & 0.832 \\
\hline H6-LR (20) & 0.60 & 1000 & 0.2708 & 0.050 & 0.0131 & 0.7292 & 0.7 & 1.0 & 256 & 256 & 0.675 \\
\hline L6 (1) & 0.60 & 500 & 0.2708 & 0.050 & 0.0131 & 0.7292 & 0.7 & 1.0 & 512 & 512 & 0.675 \\
\hline L3 (1) & 0.30 & 500 & 0.2708 & 0.050 & 0.0066 & 0.7292 & 0.7 & 1.0 & 512 & 512 & 0.752 \\
\hline L0 (1) & 0.00 & 500 & 0.2708 & 0.050 & 0.0 & 0.7292 & 0.7 & 1.0 & 512 & 0 & 0.832 \\
\hline L0-HR (1) & 0.00 & 500 & 0.2708 & 0.050 & 0.0 & 0.7292 & 0.7 & 1.0 & 768 & 0 & 0.832 \\
\hline \hline H6s8 (8) & 0.06 & 1000 & 0.2708 & 0.050 & 0.0131 & 0.7292 & 0.7 & 1.0 & 512 & 512 & 0.832 \\
\hline H0s8 (8) & 0.00 & 1000 & 0.2708 & 0.050 & 0.0 & 0.7292 & 0.7 & 1.0 & 512 & 0 & 0.675 \\
\hline H0s8-CDM (8) & 0.00 & 1000 & 0.2708 & 0.050 & 0.0 & 0.7292 & 0.7 & 1.0 & 512 & 0 & 0.701 \\
\hline L6-1 (1) & 0.60 & 500 & 0.2708 & 0.050 & 0.0131 & 0.7292 & 0.7 & 1.0 & 512 & 512 & 0.832 \\
\hline \hline L6-2 (1) & 0.60 & 500 & 0.3000 & 0.050 & 0.0131 & 0.7000 & 0.7 & 1.0 & 512 & 512 & 0.749 \\
\hline
\end{tabular}

Table 1. Summary of the simulations used in the present work. The simulations have been divided into four different groups (see text for details). The sizes of the simulations boxes are either 500 or $1000 \mathrm{~h}^{-1}$ Mpc. The name of the simulations with box sizes of $1000 h^{-1} \mathrm{Mpc}$ starts with $\mathrm{H}$ (from Huge), whereas the name of simulations with box sizes of $500 h^{-1} \mathrm{Mpc}$ begins with L (from Large). $m_{\nu_{i}}$ is the mass of a single neutrino species, and $\Sigma_{i} m_{\nu_{i}}$ is the sum of the neutrino masses. The number of independent simulations for each cosmological model is shown in parentheses after the name of each simulation.

the simulations within this group are in the value of their parameters $\Omega_{\mathrm{cdm}}, \Omega_{\nu}$ and $\sigma_{8}$. Since $\Omega_{\mathrm{m}}$ is kept constant for all the simulations within this group, simulations with higher values of $\Omega_{\nu}$ have smaller values of $\Omega_{\mathrm{cdm}}$, such as their sum is the same for all the models. The value of the parameter $\sigma_{8}$ depends on the neutrino masses since the amplitude of the power spectrum is fixed on large scales, thus, models with higher neutrino masses will have a lower value of $\sigma_{8}$. We note that within this group, the model with massless neutrinos has cosmological values in agreement with the latest results of Planck [20]. To distinguish the simulations within this group we add the suffix "Planck" to their names. The simulations within this group have been mainly used to study the clustering of galaxies.

The simulations belonging to the second group are: H6, H3, H0, H0-HR, H6-LR, L6, L3, L0, L0-HR. The characteristic of these simulations are the same as those of group 1, i.e. they share the value of the parameters $\Omega_{\mathrm{b}}, \Omega_{\Lambda}, h, n_{s}, \Omega_{\mathrm{m}}=\Omega_{\mathrm{cdm}}+\Omega_{\mathrm{b}}+\Omega_{\nu}$ and $A_{s}$ (different from group 1) and differ in their values of $\Omega_{\mathrm{cdm}}, \Omega_{\nu}$ and $\sigma_{8}$. Whereas the cosmological parameters of the simulation with massless neutrinos in the group 1 are in agreement with Planck, the massless cosmological model of group 2 is instead close to the latest results of WMAP. We have used the simulations in this group to study both the clustering of dark matter haloes and galaxies.

Simulations in group 3 share the cosmology of group 2 except for the amplitude of initial fluctuations, $A_{s}$. We have used these simulations to study the $\Omega_{\nu}-\sigma_{8}$ degeneracy, choosing $A_{s}$ to obtain models characterized by different neutrino masses and by the same value of $\sigma_{8}$. The simulations belonging to this group are: H6s8, H0s8, H0s8-CDM and L6-1, with the latter employed as well to study the impact of $\sigma_{8}$ on galaxy clustering properties.

Finally group 4 is constituted by the simulation L6-2 only. Although most of the values of the cosmological parameters of this simulation are equal to those of simulations in groups 2 and 3 , this simulation has a higher value of $\Omega_{\mathrm{m}}$. The value of the parameter $A_{s}$ is however the same as the one of the simulations in group 2. We have used this simulation to study the impact of $\Omega_{\mathrm{m}}$ on galaxy clustering.

For each simulation we identify the dark matter haloes using two different algorithms: the 
Friends-of-Friends (FoF) group finder [46] and the SUBFIND algorithm [47]. We have run the FoF algorithm with two different values of the linking length parameter: $b=0.16$ and $b=0.2$. Both algorithms have been run only on top of the CDM component. We have done this to avoid the contamination in mass arising from unbounded neutrinos. This effect can significantly bias the mass of low mass haloes. In the appendix B we show that our results, in terms of the halo-matter bias are not affected if the haloes are instead identified by using the total matter distribution. Moreover, in Paper II and Paper III we checked that running the halo finder algorithms just on top of the CDM distribution has a negligible impact on the halo mass function. We emphasize that this effect is expected since studies related with the neutrino clustering [23-26], have shown that the neutrino contribution to the total mass of the halo is below $0.5 \%$ for reasonable neutrino masses, as the ones considered here.

The dark matter haloes we have used for this paper correspond to the groups identified by SUBFIND. Those groups correspond to spherical overdensity (SO) haloes with the virial radius taken as the radius at which the mean interior density is 200 times that of the universe mean density at that redshift. We will refer to the masses of these haloes as $M_{200}$. Both algorithms, FoF and SUBFIND, provide us with the position of the center of the haloes, that we use when measuring the power spectrum or correlation function of the dark matter haloes.

\section{Clustering of dark matter haloes}

In this section we investigate the impact of massive neutrinos on the spatial distribution of dark matter haloes. We characterize the clustering of dark matter haloes by using 2-points statistics both in Fourier and in configuration space. We choose to consider both estimators, the power spectrum and the 2-pt correlation function since they probe different scales and have different sensitivity to non-linear effects and scale-dependent bias. We start by presenting the results in terms of the power spectrum in subsection 3.1, while the halo clustering in terms of the correlation function is shown in subsection 3.2. In this paper we limit our study to SO haloes, leaving the results in terms of FoF haloes to our companion paper [39].

\subsection{Halo bias: power spectrum}

It is well known that dark matter haloes, like galaxies, are biased tracers of the underlying matter density field. In this section we investigate the clustering properties of dark matter haloes in several cosmological models with different neutrino masses.

The bias between the spatial distribution of haloes and that of matter is usually computed as the ratio between the power spectrum of haloes $\left(P_{\mathrm{hh}}\right)$ to that of the matter $\left(P_{\mathrm{mm}}\right)$, that is $b_{\mathrm{hh}}^{2}(k)=$ $P_{\mathrm{hh}}(k) / P_{\mathrm{mm}}(k)$. However, this estimate is prone to stochasticity in the bias relation. For this reason we have also used the alternative estimator, $b_{\mathrm{hm}}(k)=P_{\mathrm{hm}}(k) / P_{\mathrm{mm}}(k)$, where $P_{\mathrm{hm}}(k)$ is the halomatter cross-power spectrum, which, being based on the cross power, is less sensitive to stochasticity [48-52].

The power spectrum is computed in the following way: we assign the positions of the particles or halo centers to a regular cubic grid with $N \times N \times N$ points using the cloud-in-cell (CIC) interpolation scheme. Then, we compute the value of the density contrast, $\delta(\vec{r})=\rho(\vec{r}) / \bar{\rho}-1$, at each point of the grid. By using the Fast Fourier Transform algorithm, we calculate the density contrast in Fourier space, $\delta(\vec{k})$. We correct for the CIC mass assignment scheme as [53, 54]:

$$
\delta(\vec{k}) \rightarrow \frac{\delta(\vec{k})}{W^{2}(k)},
$$

where

$$
W(k)=\left[\prod_{i=1}^{3} \frac{\sin \left(\pi k_{i} / 2 k_{N}\right)}{\left(\pi k_{i} / 2 k_{N}\right)}\right]^{2},
$$


with $k=\sqrt{k_{1}^{2}+k_{2}^{2}+k_{3}^{2}}$ and $k_{N}=\pi N / L$ being the Nyquist wave number for the chosen grid $(L$ is the size of the simulation box). Finally, the power spectrum is computed by averaging modes with wave numbers within narrow intervals $k-\triangle k / 2<|\vec{k}|<k+\triangle k / 2$

$$
P(k)=\frac{1}{\mathrm{~N}_{\text {modes }}} \sum_{\vec{k} \in k} \delta(\vec{k}) \delta^{*}(\vec{k}),
$$

where $\mathrm{N}_{\text {modes }}$ is the number of modes in the interval over which $P(k)$ is computed. In practice, we compute the power spectrum in bins of size $2 \pi / L$, using a grid with $N=512$.

The previous procedure is used to compute the power spectrum of the CDM particles, the neutrinos and the dark matter haloes. When the computational box contains more than one mass component, e.g. CDM and neutrinos, the power spectrum of the total mass is obtained from the following density field:

$$
\delta_{\mathrm{m}}(\vec{r})=\frac{\Omega_{\mathrm{cdm}}}{\Omega_{\mathrm{cdm}}+\Omega_{\nu}} \delta_{\mathrm{cdm}}(\vec{r})+\frac{\Omega_{\nu}}{\Omega_{\mathrm{cdm}}+\Omega_{\nu}} \delta_{\nu}(\vec{r}),
$$

where $\delta_{\mathrm{cdm}}(\vec{r})$ and $\delta_{\nu}(\vec{r})$ are the values of the density contrast in the mesh points for the CDM and the neutrinos respectively. For the cross-spectrum of two different fluids we compute the values of density contrast in Fourier space for each fluid, $\delta_{1}(\vec{k})$ and $\delta_{2}(\vec{k})$, correcting for the CIC procedure as outlined above, and we compute their cross-spectrum by calculating:

$$
P_{12}(k)=\frac{1}{\mathrm{~N}_{\text {modes }}} \sum_{\vec{k} \in k} \operatorname{Re}\left[\delta_{1}^{*}(\vec{k}) \delta_{2}(\vec{k})\right] .
$$

The measurements of the auto-power and cross-power spectra may require a correction to account for the discreteness nature of the objects used to compute them. If so, the measured halo power spectrum will be shifted with respect to the correct one by the inverse of the halo number density, $\bar{n}_{\text {haloes}}^{-1}$, on all scales (this is called white noise, since it is independent of scale). We have corrected our power spectrum measurements to account for this effect. The details of this correction for the different cases can be found on the appendix A.

We use the simulations $\mathrm{H} 0, \mathrm{H} 3$ and $\mathrm{H} 6$ to study the impact of neutrino masses on the spatial distribution of dark matter haloes. Those simulations have the same cosmological parameter values, with the exception of $\Omega_{\nu}, \Omega_{\mathrm{cdm}}$ and $\sigma_{8}$. The value of $\Omega_{\mathrm{m}}=\Omega_{\mathrm{cdm}}+\Omega_{\mathrm{b}}+\Omega_{\mathrm{b}}+\Omega_{\nu}$ is held fixed at 0.2708 for the three simulations, therefore, by comparing the results among the different models, we are comparing universes in which the same dark matter content is made up by different fractions of massive neutrinos. Moreover, the amplitude of the power spectrum is fixed on large scales, implicitly assuming that those scales are very well constrained by the CMB data. This constraint implies that the value of $\sigma_{8}$ depends on the neutrino masses, in such a way that the larger the neutrino masses the lower the value of $\sigma_{8}$. Each simulation consists of 8 independent realizations of the same density field, obtained by using a different seed when computing the initial conditions. For each realization, we select the dark matter haloes with masses, $M_{200}$, above $2 \times 10^{13} h^{-1} \mathrm{M}_{\odot}$ and $4 \times 10^{13} h^{-1} \mathrm{M}_{\odot}$ and compute the haloes power spectrum, the haloes-dark matter cross-power spectrum, the matter-matter auto-power spectrum and the bias: $b_{\mathrm{hm}}(k)=P_{\mathrm{hm}}(k) / P_{\mathrm{mm}}(k)$ and $b_{\mathrm{hh}}^{2}(k)=P_{\mathrm{hh}}(k) / P_{\mathrm{mm}}(k)$. We have selected the dark matter haloes with those masses to, on one hand, maximize our statistics while, on the other hand, being able to investigate whether our results are sensitive to the halo masses.

In Fig. 1 we show, for each cosmological model, the value of $b_{\mathrm{hm}}(k)$, averaged over the 8 realizations and its r.m.s. scatter (errorbars). The blue points represent the results for the $\Sigma_{i} m_{\nu_{i}}$ $=0.6 \mathrm{eV}$ cosmology, whereas the green and red points are for the $\Sigma_{i} m_{\nu_{i}}=0.3$ and $\Sigma_{i} m_{\nu_{i}}=0.0$ $\mathrm{eV}$ cosmologies, respectively. The results are shown at $z=0, z=0.5$ and $z=1$ for the two mass thresholds: $2 \times 10^{13} h^{-1} \mathrm{M}_{\odot}$ (left column) and $4 \times 10^{13} h^{-1} \mathrm{M}_{\odot}$ (right column). We do not show the results at $z=2$ since the number of haloes above threshold is small and results are very noisy.

We find that at any given wave number, the bias increases with the neutrino masses. The different value of the parameter $\sigma_{8}$ in the three cosmological models considered is the main reason of this effect. Objects with masses larger than $2 \times 10^{13} h^{-1} \mathrm{M}_{\odot}$ are rarer in a cosmology with $\sigma_{8}=0.675$ 

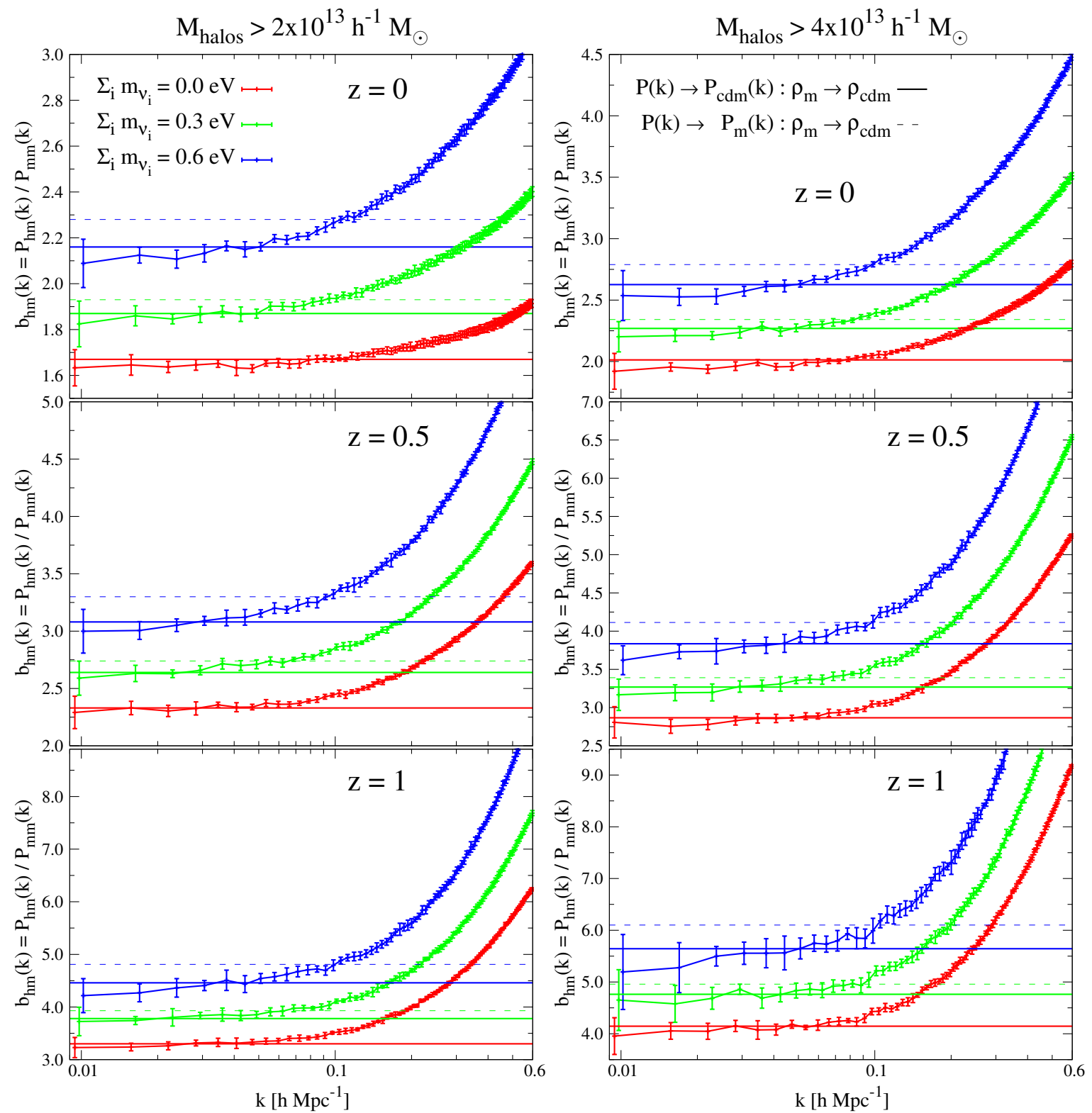

Figure 1. Halo-matter bias, computed as the ratio of the halo-matter cross-power spectrum to the matter power spectrum, for massless and massive neutrino simulations. We show the bias for haloes with masses, $M_{200}$, larger than $2 \times 10^{13} h^{-1} \mathrm{M}_{\odot}$ (left column) and for haloes with masses larger than $4 \times 10^{13} h^{-1} \mathrm{M}_{\odot}$ (right column) at redshifts $z=0, z=0.5$ and $z=1$ (as indicated on each panel). The results are shown for three different cosmologies with the same value of $\Omega_{\mathrm{m}}=0.2708$ and $A_{s}$ but different neutrino masses: $\Sigma_{i} m_{\nu_{i}}=0.0 \mathrm{eV}$ (red), $\Sigma_{i} m_{\nu_{i}}=0.3 \mathrm{eV}$ (green) and $\Sigma_{i} m_{\nu_{i}}=0.6 \mathrm{eV}$ (blue). The points and the error bars represent the mean value and the standard deviation from the set of 8 independent realizations comprising each cosmological model. The horizontal lines show the value of the bias on large scales as predicted by the Tinker [55] fitting formula. We have used two different prescriptions when using that formula: the matter prescription (thin dashed lines), consisting in using the linear power spectrum for the whole matter and the cold dark matter prescription (thick solid lines), in which we use the linear matter power spectrum of the CDM component. In both prescriptions we take $\rho_{\text {cdm }}$ as the value of $\rho_{\mathrm{m}}$. Note that the results for the $\Sigma_{i} m_{\nu_{i}}$ $=0.3 \mathrm{eV}$ and $\Sigma_{i} m_{\nu_{i}}=0.6 \mathrm{eV}$ cosmologies have been slightly displaced in the $x$-axis for clarity. 
(as in the cosmology with $\Sigma_{i} m_{\nu_{i}}=0.6 \mathrm{eV}$ ) than in a cosmology with $\sigma_{8}=0.832$ (as in the massless neutrino cosmology), and thus, the bias is expected to be higher in the former model than in the latter [37]. On large scales, we find that the bias flattens out for all the cosmological models. However, we find that even on large scales the bias displays some weak scale-dependence for the simulations with massive neutrinos. We will discuss this point in detail below.

We check whether our results on large scales can reproduced by the Tinker fitting formula [55]

$$
b_{\text {Tinker }}(\nu)=1-A \frac{\nu^{a}}{\nu^{a}+\delta_{c}^{a}}+B \nu^{b}+C \nu^{c},
$$

with $A=1.0+0.24 y e^{-(4 / y)^{4}}, a=0.44 y-0.88, B=0.183, b=1.5, C=0.019+0.107 y+0.19 e^{-(4 / y)^{4}}$ and $c=2.4$, being $y=\log _{10} \triangle$. $\triangle$ is the halo mean overdensity within its virial radius in units of the mean matter density of the universe, which is set to 200 for this paper. In the Tinker fitting formula, the dependence of the bias on the halo mass is parametrized in terms of the peak height of the linear density field: $\nu=\delta_{c} / \sigma(M)$, where $\delta_{c}=1.686^{6}$ is the value of the linearly extrapolated overdensity at the time of collapse and $\sigma(M)$ is defined as

$$
\sigma^{2}(M)=\frac{1}{2 \pi^{2}} \int_{0}^{\infty} P_{\operatorname{lin}}(k, z) \hat{W}(k, R) k^{2} d k
$$

where $P_{\text {lin }}(k, z)$ is the linear matter power spectrum at redshift $z$ and $\hat{W}(k, R)$ is the Fourier transform of the top-hat window function of radius $R$. The relationship between the halo mass and the variable $R$ is given by $M=4 \pi \rho_{\mathrm{m}} R^{3} / 3$, with $\rho_{\mathrm{m}}$ being the mean matter density of the universe.

When using the Tinker fitting formula we need to specify both the linear power spectrum $P_{\text {lin }}(k)$ and $\rho_{\mathrm{m}}$; the latter relates the radius in the top-hat window function to the halo mass, whereas the former enters in the definition of $\sigma(M)$. For cosmologies with massless neutrinos the linear power spectrum of the cold dark matter and that of the total matter coincide. However, when more components contribute to $\Omega_{\mathrm{m}}$ it is not clear which power spectrum is more relevant to describe halo clustering and their abundance. Recent studies related to the impact of massive neutrinos on the halo mass function $[24,26,37]$ have suggested that a reasonable agreement between theory and simulations is achieved if the total matter linear power spectrum is used, together with $\rho_{\mathrm{m}}=\rho_{\text {cdm }}$ (used to establish the M-R relationship). In this paper we use that prescription for massive neutrinos cosmologies, that we will refer to as the matter prescription, when computing the value of the bias and the halo mass function. Moreover, we also test the cold dark matter prescription, which uses the linear power spectrum of the CDM component together with $\rho_{\mathrm{m}}=\rho_{\mathrm{cdm}}$, as suggested by [56] and tested in our companion papers $[39,40]$ agains N-body simulations for studies of the halo mass functions in universes with massive neutrinos.

We compute the linear power spectrum for the CDM component through CAMB as:

$$
P_{\operatorname{lin}}^{\mathrm{cdm}}(k)=\left(\frac{T_{\mathrm{cdm}}}{T_{\mathrm{m}}}\right)^{2} P_{\mathrm{lin}}^{\mathrm{m}}(k),
$$

where the $T_{\mathrm{cdm}}(k)^{7}$ and $T_{\mathrm{m}}(k)$ are the transfer functions for the CDM and the total matter respectively. To compare our results with the fitting formula of Tinker, we need to calculate the effective bias for the haloes we selected in our simulations, i.e. for haloes with masses above a certain mass

$$
\bar{b}(z)=\frac{\int_{M_{\min }}^{M_{\max }} b_{\text {Tinker }}(M, z) n(M, z) d M}{\int_{M_{\min }}^{M_{\max }} n(M, z) d M},
$$

where $M_{\min }$ and $M_{\max }$ are the minimum and maximum masses of the dark matter haloes used in the analysis. The halo mass function, $n(M, z) d M$, specifies the comoving number density of dark matter

\footnotetext{
${ }^{6}$ We have neglected the dependence of this parameter with cosmology and with the masses of the neutrinos since that is very weak.

${ }^{7}$ In our case we incorporate the baryons effects into the CDM particles when generating the initial conditions by setting: $T_{\mathrm{cdm}}(k)=\left[\Omega_{\mathrm{cdm}} T_{\mathrm{cdm}}(k)+\Omega_{\mathrm{b}} T_{\mathrm{b}}(k)\right] /\left(\Omega_{\mathrm{cdm}}+\Omega_{\mathrm{b}}\right)$, where $T_{\mathrm{cdm}}(k)$ and $T_{\mathrm{b}}(k)$ are directly obtained via CAMB.
} 
haloes with masses within the interval $[M, M+d M]$. For the halo mass function we use the fitting formula of Tinker et al. 2008 [42] with the same prescriptions as above for cosmologies with massive neutrinos, i.e. $\sigma(M)$ is computed using the total matter linear power spectrum when using the matter prescription, whereas the linear CDM power spectrum is used for the cold dark matter prescription. In both prescriptions we use $\rho_{\mathrm{cdm}}$ as the value of $\rho_{\mathrm{m}}$. In [40] we show that the halo mass function of SO haloes in cosmologies with massive neutrinos is well reproduced by the Tinker fitting formula along with the cold dark matter prescription. We set $M_{\max }=3 \times 10^{15} h^{-1} \mathrm{M}_{\odot}$. We have explicitly checked that our results do not significantly change if we choose a different (but reasonable) value of $M_{\max }$.

In Fig. 1 the horizontal, thin dashed line shows the prediction of Tinker's bias obtained using the matter prescription, as opposed to the thick, solid horizontal lines that refer to the cold dark matter prescription. We find that, at all redshifts and for the two different mass thresholds, the bias on large scales is better reproduced if we use the Tinker fitting formula with the cold dark matter prescription for massive neutrinos. This is more clearly seen in the results for the $\Sigma_{i} m_{\nu_{i}}=0.6 \mathrm{eV}$ cosmology, where the value of the bias on large scales computed using the matter prescription is shifted to higher values than the ones obtained from the N-body simulations. This point is investigated more extensively in our companion paper [39].

In Fig. 2 we show the results when the bias is computed using the definition $b_{\mathrm{hh}}^{2}(k)=P_{\mathrm{hh}}(k) / P_{\mathrm{mm}}(k)$. In this case as well we find that the bias of the dark matter haloes increases with the neutrino masses, as a consequence of the different values of the parameter $\sigma_{8}$ for the models studied here. In contrast to the results of the bias computed using the halo-matter cross-power spectrum, $b_{\mathrm{hm}}(k)$, we find that the value of bias, $b_{\mathrm{hh}}(k)$, drops on small scales. This is due to the halo-exclusion effect: the probability of finding two dark matter haloes on very small scales shrinks quickly, as a consequence of the finite size of those. We also find that the Tinker fitting formula reproduces reasonably well our results, in terms of the bias on large scales when the cold dark matter prescription for cosmologies with massive neutrinos is used. The disagreement between our results and the Tinker fitting formula is larger is the latter is used along the matter prescription. However, we note that the agreement between our results on large scales and the Tinker fitting formula plus the cold dark matter prescription is poorer than when the bias is computed as $b_{\mathrm{hm}}(k)=P_{\mathrm{hm}}(k) / P_{\mathrm{mm}}(k)$. We also find this effect when the bias is measured using the correlation function (see the following subsection). The scale-dependent bias on large scales is clearly seen in the models with massive neutrinos, being more prominent at high redshift and for the model with $\Sigma_{i} m_{\nu_{i}}=0.6 \mathrm{eV}$ neutrinos.

We now focus on the $\Omega_{\nu}-\sigma_{8}$ degeneracy. Our purpose here is to investigate how the spatial distribution of dark matter haloes is affected by $\Sigma_{i} m_{\nu_{i}}$ for cosmological models that share the same value of the cosmological parameter $\sigma_{8}$. To answer this question, we compare the bias computed from the simulations $\mathrm{H} 0$ and $\mathrm{H} 6$ with the bias measured in the simulations $\mathrm{H} 6 \mathrm{~s} 8$ and $\mathrm{H} 0 \mathrm{~s} 8$. The former represents a cosmology with the same value of $\sigma_{8}$ as the simulation $\mathrm{H} 0$, but it also contains neutrinos with masses equal to $\Sigma_{i} m_{\nu_{i}}=0.6 \mathrm{eV}$, whereas the latter corresponds to a cosmology with massless neutrinos but with the same value of $\sigma_{8}$ as the simulation H6. In Fig. 3 we show the results, in terms of $b_{\mathrm{hm}}(k)$, together with the Tinker prediction using the cold dark matter prescription for massive neutrinos. The error bars represent the standard deviation around the mean value from the set of 8 realizations available for each cosmological model. We find that the bias for simulations that share the same value of $\sigma_{8}$ are very similar, though not exactly the same. On large scales, our results are well reproduced by the fitting formula of Tinker along with the cold dark matter prescription for models with massive neutrinos. For models with the same value of $\sigma_{8}$ the Tinker fitting formula predicts a difference on the bias of $\sim 3 \%$ at $z=0$ while this number increases up to $\sim 8 \%$ at $z=2$. It is not surprising that the differences between models that share the same value of $\sigma_{8}$ increase with redshift, since their growth factors are different. Given these results we conclude that the $\Omega_{\nu}-\sigma_{8}$ degeneracy (in terms on the bias on large scales) is not perfect. In terms of the Tinker fitting formula, the value of large-scale bias will be degenerate in $\Sigma_{i} m_{\nu_{i}}$ for models that share the same linear CDM power spectrum. We further discuss this point in our companion paper [39].

The large scale dependence of the bias on scales $k<0.1 h \mathrm{Mpc}^{-1}$ in presence of massive neutrino deserves some additional investigation. No such dependence is found when considering massless 

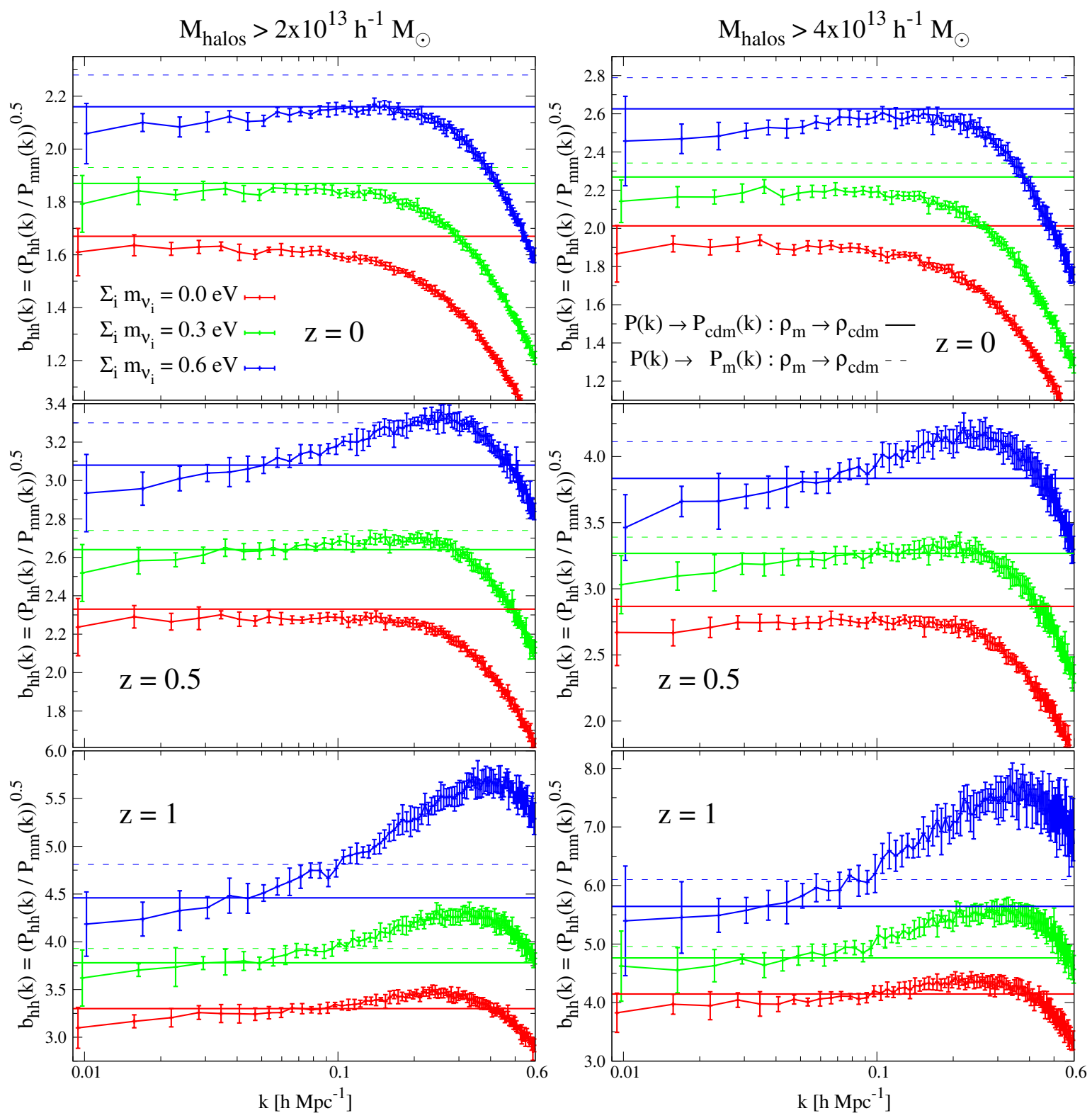

Figure 2. Same as Fig. 1 but with the bias computed as $b_{\mathrm{hh}}^{2}(k)=P_{\mathrm{hh}}(k) / P_{\mathrm{mm}}(k)$.

neutrinos (H0 and H0s8 simulations), as expected. We emphasize this by showing with solid lines in Fig. 4 the bias, $b_{\mathrm{hm}}(k)$, for the different cosmological models normalized to the bias of the model with massless neutrinos and $\sigma_{8}=0.832$ (simulation $\mathrm{H} 0$, reference model). The ratios are shown at redshifts $z=0, z=0.5$ and $z=1$ for the simulations H6 (blue), H3 (green), H6s8 (black) and H0s8 (magenta). The horizontal dotted lines represent the Tinker prediction along with the cold dark matter prescription for massive neutrinos. Focusing on the results of the simulations H3 and H6 (sharing the same value of $A_{s}$ as the reference model), we find a clear scale-dependent bias trend on large scales. If we compare the results of the simulation H0s8 (magenta) with the one of the simulation $\mathrm{H} 6$ (blue), both sharing the same value of the parameter $\sigma_{8}$, we find that for the former, the bias is constant for $k \lesssim 0.1 \mathrm{~h} \mathrm{Mpc}^{-1}$, whereas we find a clear scale-dependent bias in the simulation with $\Sigma_{i} m_{\nu_{i}}=0.6 \mathrm{eV}$ neutrinos (H6). In our companion paper [39], we show that this effect is due to the 

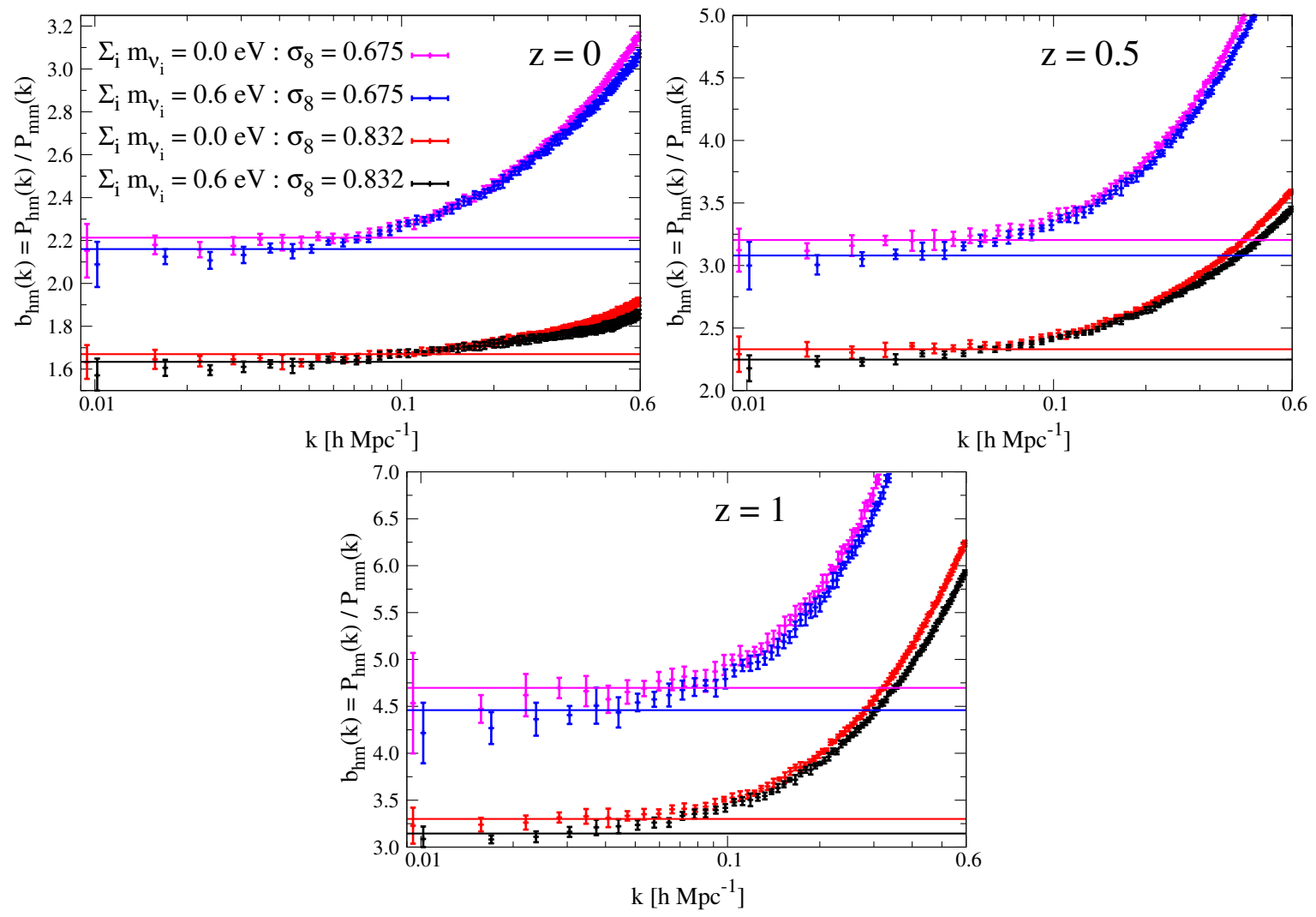

Figure 3. $\Omega_{\nu}-\sigma_{8}$ degeneracy in the halo-matter bias. We plot the bias, $b_{h m}$, between the spatial distribution of haloes with masses, $M_{200}$, above $2 \times 10^{13} h^{-1} \mathrm{M}_{\odot}$, and this of the underlying dark matter, comparing different cosmologies with the same value of $\sigma_{8}$ but different $\Sigma_{i} m_{\nu_{i}}$. The bias is shown at three different redshifts: $z=0$ (top-left), $z=0.5$ (top-right) and $z=1$ (bottom). Models with a value of $\sigma_{8}=0.675$ are represented by the magenta and the blue points, which have $\Sigma_{i} m_{\nu_{i}}=0 \mathrm{eV}$ and $\Sigma_{i} m_{\nu_{i}}=0.6 \mathrm{eV}$ respectively. The red and black lines correspond to models with $\Sigma_{i} m_{\nu_{i}}=0 \mathrm{eV}$ and $\Sigma_{i} m_{\nu_{i}}=0.6 \mathrm{eV}$ respectively, with the same value of $\sigma_{8}=0.832$. The horizontal lines show the prediction of the bias on large scales using the Tinker formula plus the cold dark matter prescription for neutrinos. The error bars are the dispersion around the mean bias value from the set of 8 independent realizations comprising each simulation.

presence of massive neutrinos, and that only by calculating the bias as the ratio $P_{\mathrm{hc}}(k) / P_{\mathrm{cc}}(k)$ where $P_{\mathrm{hc}}(k)$ and $P_{\mathrm{cc}}(k)$ are the haloes-cold dark matter and cold dark matter power spectrum respectively, the bias becomes scale-independent, and universal, on large scales. We have computed the bias using $P_{\mathrm{hc}}(k) / P_{\mathrm{cc}}(k)$ for the models with massive neutrinos, and we show the results of the ratio with respect to the reference model in Fig. 4 with dashed lines. On large scales $\left(k \lesssim 0.1 h^{-1} \mathrm{Mpc}\right)$ we find that the scale-dependence of the bias is highly suppressed if quantities are computed with respect to the CDM distribution instead of over the total matter distribution.

\subsection{Halo bias: correlation function}

In this subsection we further investigate the clustering properties of dark matter haloes in cosmologies with massive neutrinos using the correlation function statistics, i.e. the Fourier transform of the power spectrum. While the information content is in principle the same as with the power spectra, the use of a different estimate allows to emphasize clustering properties on different scales. The purpose of this section is, thus, to corroborate the results obtained in the previous section.

As we have done in the above subsection with the power spectrum technique, we study the impact of massive neutrinos on the bias between the spatial distribution of dark matter haloes 

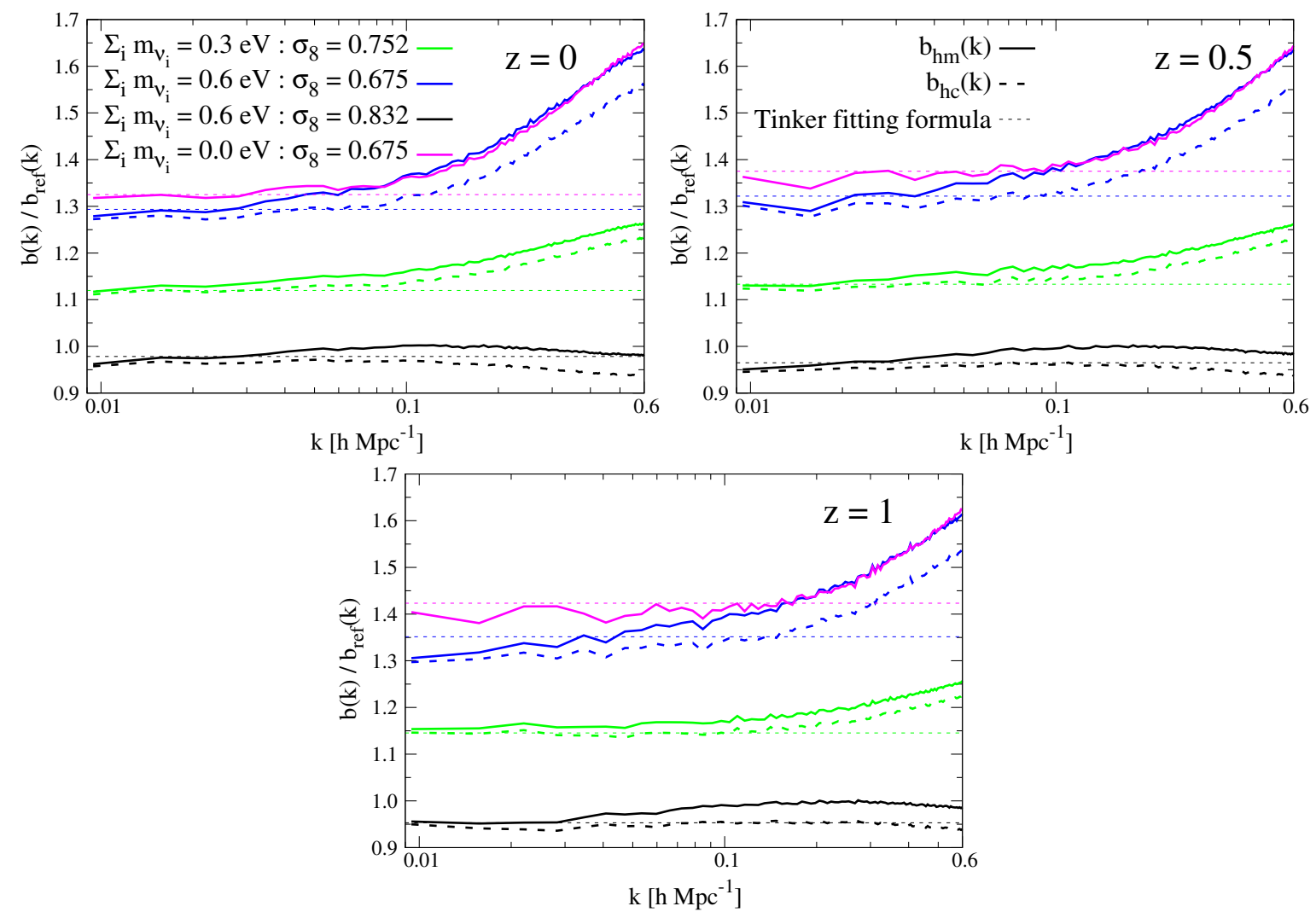

Figure 4. Bias ratio for different cosmological models. We show the bias of dark matter haloes with masses above $2 \times 10^{13} h^{-1} \mathrm{M}_{\odot}$ for the simulations H6 (blue), H3 (green), H6s8 (black) and H0s8 (magenta) normalized by the bias of the simulation H0 (reference model). The results are shown at four different redshifts, whose value is indicated on each panel. The solid lines show the results when the bias is computed using the total matter distribution, i.e. CDM plus massive neutrinos, whereas the dashed lines represent the results when the bias is calculated by using just the CDM distribution. In our companion paper [39], we show that only by computing the bias with respect to the cold dark matter distribution, the bias on large scale becomes scale-independent and universal.

and that of the underlying matter using two different estimators: $b_{\mathrm{hh}}^{2}(r)=\xi_{\mathrm{hh}}(r) / \xi_{\mathrm{mm}}(r)$ and $b_{\mathrm{hm}}(r)=\xi_{\mathrm{hm}}(r) / \xi_{\mathrm{mm}}(r)$, where $\xi_{\mathrm{hh}}(r), \xi_{\mathrm{hm}}(r)$ and $\xi_{\mathrm{mm}}(r)$ are the 2-pt autocorrelation functions of the dark matter haloes, the haloes-matter cross-correlation function and the matter 2-pt autocorrelation function, respectively.

Our halo catalogue consists of all the dark matter haloes with masses, $M_{200}$, above $2 \times 10^{13} h^{-1} \mathrm{M}_{\odot}$, as given by the SUBFIND groups. The matter 2-pt autocorrelation function and the haloes-matter cross-correlation function is not measured using all the dark matter particles (i.e. CDM particles plus neutrinos) since the computational cost of such calculation is prohibitively large. We however decide to randomly select 5 million particles of both types. We have checked that our results are robust to this choice in the sense that increasing the number of randomly selected particles by a factor of 2 does not significantly change them.

The 2-pt autocorrelation functions are computed using the Landy-Szalay estimator [57]:

$$
\xi(r)=\frac{\mathrm{DD}(r)-2 \mathrm{DR}(r)+\mathrm{RR}(r)}{\mathrm{RR}(r)} .
$$

Here $\mathrm{DD}(r)$ and $\mathrm{RR}(r)$ are the normalized number of pairs with distances in the interval $[r, r+$ $d r]$, in the initial catalogue (data-data) and in the so-called random catalogue (random-random) 

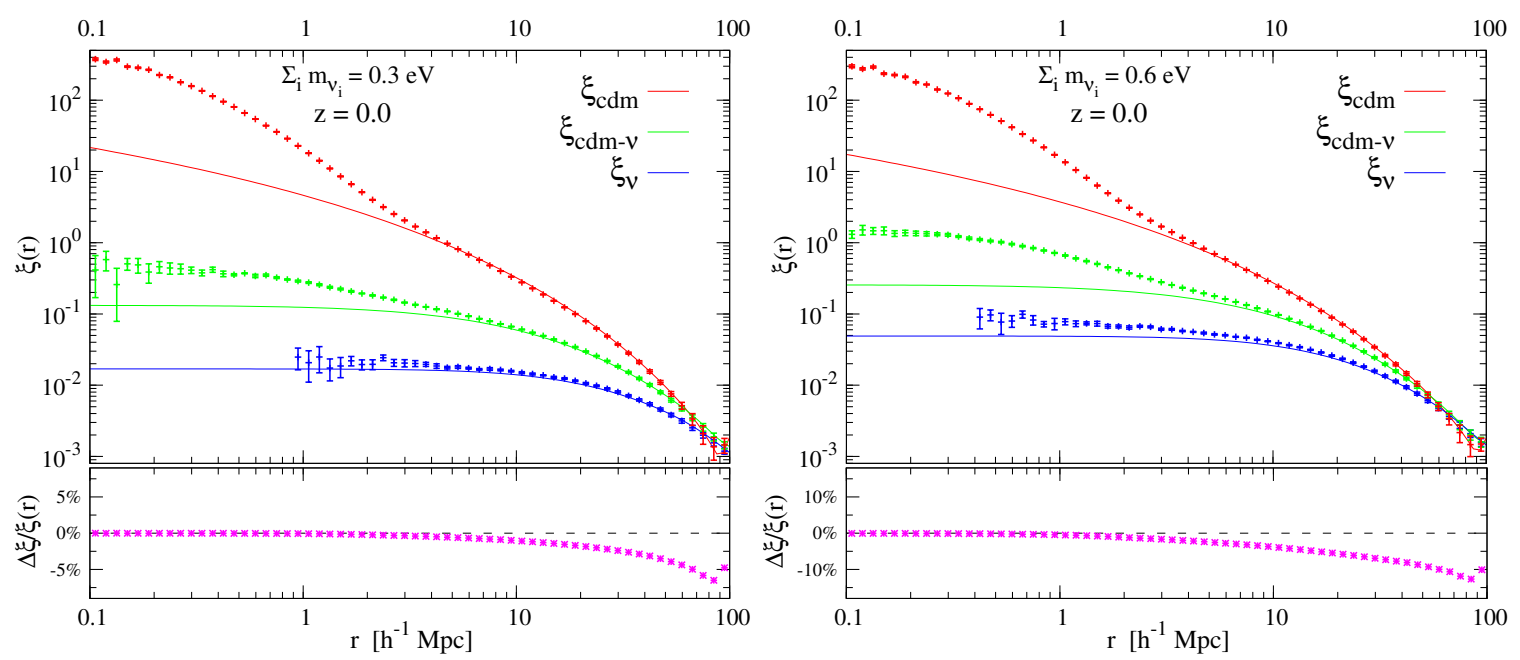

Figure 5. The panels show the 2-pt autcorrelation function of the CDM (red), the neutrinos (blue) and their cross-correlation (green) for the cosmologies with $\Sigma_{i} m_{\nu_{i}}=0.3 \mathrm{eV}$ (left panel) and $\Sigma_{i} m_{\nu_{i}}=0.6 \mathrm{eV}$ (right panel) at $z=0$. The thin solid lines correspond to the linear theory prediction obtained from CAMB (see text for details). The bottom panels show the relative difference between computing the correlation function for the entire matter with and without the last two term in the right hand side of Eq. 3.12, i.e. the contribution of massive neutrinos to the total matter correlation function.

respectively, whereas $\mathrm{DR}(r)$ represents the normalized number of cross-pairs between both catalogues (data-random). We use the above estimator to compute the 2-pt autocorrelation functions of the dark matter haloes, the CDM particles and the neutrinos.

The cross-correlation function between objects of type A and objects of type B is computed using the following estimator [58]:

$$
\xi_{\mathrm{AB}}(r)=\frac{\mathrm{D}_{\mathrm{A}} \mathrm{D}_{\mathrm{B}}(r)-\mathrm{D}_{\mathrm{A}} \mathrm{R}(r)-\mathrm{D}_{\mathrm{B}} \mathrm{R}(r)+\mathrm{RR}(r)}{\mathrm{RR}(r)},
$$

where $\mathrm{D}_{\mathrm{A}} \mathrm{D}_{\mathrm{B}}(r), \mathrm{D}_{\mathrm{A}} \mathrm{R}(r), \mathrm{D}_{\mathrm{B}} \mathrm{R}(r)$ and $\mathrm{RR}(r)$ are the normalized number of type A objects-type $\mathrm{B}$ objects, type A objects-random, type B objects-random and random-random pairs with distances in the interval $[r, r+d r]$. We use this estimator to compute the CDM-neutrino, CDM-haloes and neutrino-haloes cross correlation functions.

In both estimators the random catalogue is constructed by filling the simulation box with 10 million particles randomly distributed within the interior volume. We have explicitly checked that our results do not change if we increase the number of random points when constructing the random catalogue. When computing the distances among pairs, we have taken into account the periodic boundary conditions of the simulation box.

If the N-body simulation contains more than one particle type, i.e. CDM and neutrinos, we compute the correlation function of the total matter distribution by calculating

$$
\xi_{\mathrm{m}}(r)=\left(\frac{\Omega_{\mathrm{cdm}}}{\Omega_{\mathrm{m}}}\right)^{2} \xi_{\mathrm{cdm}}(r)+\left(\frac{\Omega_{\nu}}{\Omega_{\mathrm{m}}}\right)^{2} \xi_{\nu}(r)+\left(\frac{2 \Omega_{\mathrm{cdm}} \Omega_{\nu}}{\Omega_{\mathrm{m}}^{2}}\right) \xi_{\mathrm{cdm}-\nu}(r)
$$

being $\Omega_{\mathrm{m}}=\Omega_{\mathrm{cdm}}+\Omega_{\mathrm{b}}+\Omega_{\nu} . \xi_{\mathrm{cdm}}(r)$ and $\xi_{\nu}(r)$ are the cold dark matter and neutrino 2-pt autocorrelation functions and $\xi_{\mathrm{cdm}-\nu}(r)$ is the cross-correlation among them. The haloes-matter cross-correlation function in simulations with massive neutrinos is computed as

$$
\xi_{\mathrm{hm}}(r)=\left(\frac{\Omega_{\mathrm{cdm}}}{\Omega_{\mathrm{m}}}\right) \xi_{\mathrm{hc}}(r)+\left(\frac{\Omega_{\nu}}{\Omega_{\mathrm{m}}}\right) \xi_{\mathrm{h} \nu}(r)
$$



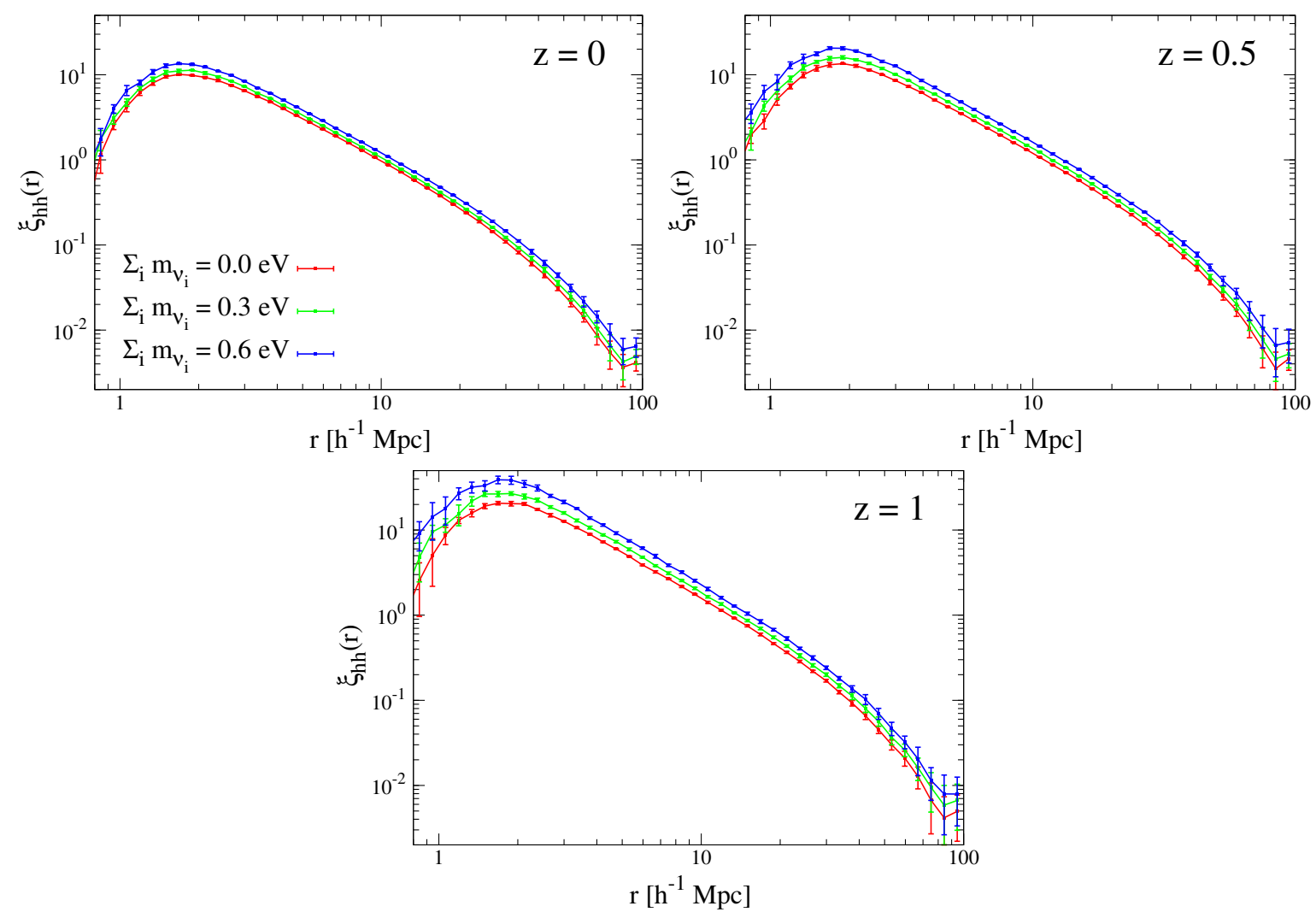

Figure 6. Dark matter haloes autocorrelation function. We show the correlation function of dark matter haloes with masses, $M_{200}$, larger than $2 \times 10^{13} h^{-1} \mathrm{M}_{\odot}$ for three cosmological models: $\Sigma_{i} m_{\nu_{i}}=0.0 \mathrm{eV}$ (red), $\Sigma_{i} m_{\nu_{i}}=0.3 \mathrm{eV}$ (green) and $\Sigma_{i} m_{\nu_{i}}=0.6 \mathrm{eV}$ (blue). Results are displayed at $z=0$ (top-left), $z=0.5$ (top-right) and $z=1$ (bottom). The points and error bars represent the mean and the dispersion around the mean obtained from the set of 8 independent realizations comprising each simulation.

with $\xi_{\mathrm{hc}}(r)$ and $\xi_{\mathrm{h} \nu}(r)$ being the haloes-CDM and the haloes-neutrinos cross-correlation functions computed using the estimator 3.11.

We now study the impact of massive neutrinos on the bias between the spatial distribution of dark matter haloes and that of the underlying matter using the models $\mathrm{H} 0, \mathrm{H} 3$ and $\mathrm{H} 6$ sharing the value of $\Omega_{\mathrm{m}}$ and $A_{s}$, i.e. models in which neutrinos made up a different fraction of the same dark matter content and models in which the amplitude of the power spectrum is fixed on large scales (error bars from CMB measurements are small on those scales). For each realization we measure the 2-pt autocorrelation functions of the dark matter haloes, the CDM particles, the neutrinos and the total matter, together with the CDM-neutrinos, CDM-haloes, neutrino-haloes and matter-haloes cross-correlation functions using the above method and estimators.

In Fig. 5 we show the 2-pt autocorrelation function of the CDM and neutrino components together with their cross-correlation function in real space, at $z=0$, for the cosmologies with $\Sigma_{i} m_{\nu_{i}}$ $=0.3 \mathrm{eV}$ (left panel) and $\Sigma_{i} m_{\nu_{i}}=0.6 \mathrm{eV}$ (right panel). The points represent the mean over the 8 independent realizations while the error bars are the standard deviation around the mean. The thin solid lines represent the linear (cross-)correlation function of each object, obtained from the linear power spectrum [as given by CAMB. For each object, its linear power spectrum has been computed using the CAMB transfer functions, and their correlation functions are calculated as follows:

$$
\xi(r)=\frac{1}{2 \pi^{2}} \int_{0}^{\infty} P_{\text {lin }}(k) \frac{\sin (k r)}{k r} k^{2} d k
$$



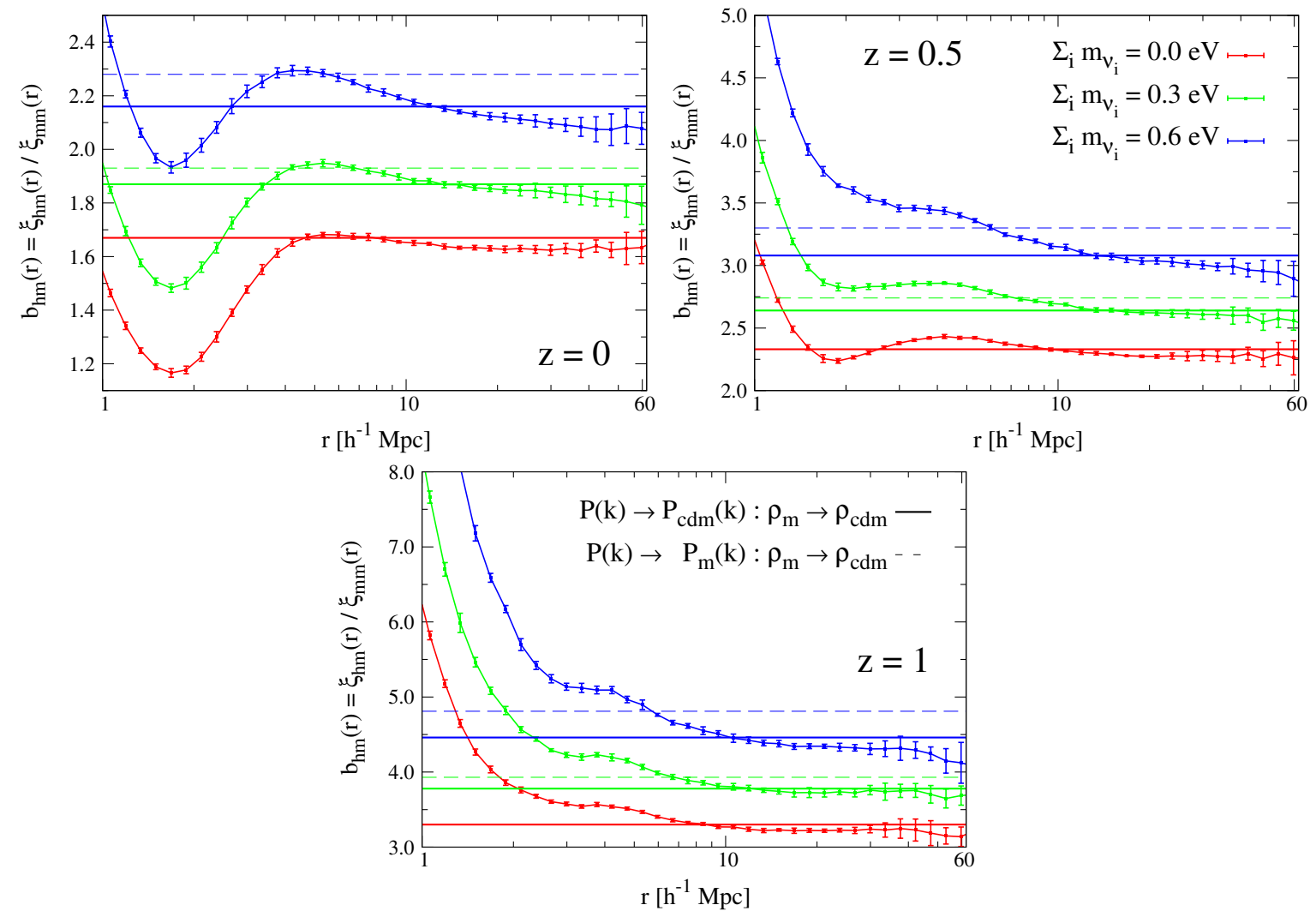

Figure 7. Bias between the spatial distribution of dark matter haloes and this of the underlying matter, computed as $b_{\mathrm{hm}}(r)=\xi_{\mathrm{hm}}(r) / \xi_{\mathrm{mm}}(r)$. We show the results at $z=0$ (left), $z=0.5$ (middle) and $z=1$ (blue) for cosmological models that share the value of $\Omega_{\mathrm{m}}$ and $A_{s}$, but have different values of $\Omega_{\nu}$. The results for the $\Sigma_{i} m_{\nu_{i}}=0 \mathrm{eV}$ are shown in red, whereas the results for the models with $\Sigma_{i} m_{\nu_{i}}=0.3 \mathrm{eV}$ and $\Sigma_{i} m_{\nu_{i}}=0.6 \mathrm{eV}$ are displayed in green and blue respectively. On large scales the bias flattens out, although we find the bias to be scale-dependent for the models with massive neutrinos. The horizontal lines represent the value of the bias on large scales obtained by using the Tinker fitting formula along with the cold dark matter prescription (solid lines) and the matter prescription.

We find that on scales larger than $\sim 20 h^{-1} \mathrm{Mpc}$ the correlation function of each tracer (CDM, neutrinos or CDM-neutrinos) is well described by the linear one. As expected, on small scales the nonlinear evolution of the CDM density field is much stronger correlated with itself than the neutrinos are. Their cross-correlation function indicates that neutrinos are certainly correlated with the CDM density field (see for instance [26]). We also explore how important are the corrections induced by neutrinos on the total matter correlation function. We compute the matter 2-pt autocorrelation function using $\xi_{\mathrm{m}}(r)=\left(\Omega_{\mathrm{cdm}} / \Omega_{\mathrm{m}}\right)^{2} \xi_{\mathrm{cdm}}(r)$, i.e. neglecting the neutrino contribution, and compared it with the estimate from Eq. 3.12. In the lower panels of Fig. 5 we plot the relative difference between these quantities. We find that on scales smaller than $\sim 5 h^{-1} \mathrm{Mpc}$ the contribution from neutrinos is indeed negligible. This happens because on those scales the correlation function is dominated by the 1-halo term, i.e. by the clustering of matter within CDM haloes, and it is well known (see [22-24, 26]) that neutrinos are significantly less clustered than CDM on these scales. On the other hand, the contribution of neutrinos, and in particular of the CDM-neutrino cross-correlation function, becomes more important on large scales. At $r \sim 80 h^{-1} \mathrm{Mpc}$, the neutrino contribution to the total matter correlation function is larger than $5 \%$ and $10 \%$ for neutrinos with $\Sigma_{i} m_{\nu_{i}}=0.3 \mathrm{eV}$ and $\Sigma_{i} m_{\nu_{i}}=0.6$ $\mathrm{eV}$ respectively.

In Fig. 6 we show the autocorrelation function of the dark matter haloes with masses, $M_{200}$, 

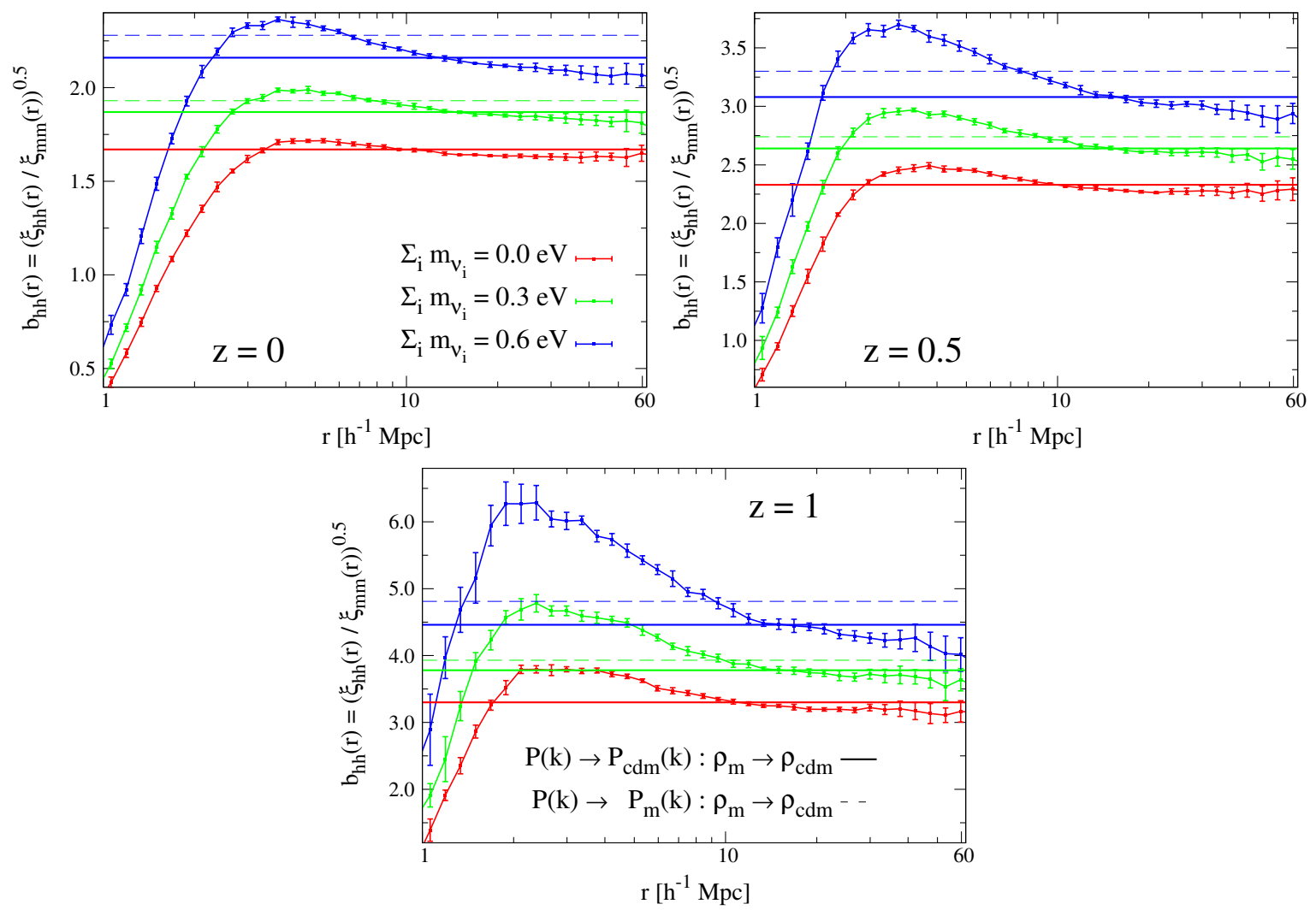

Figure 8. Same as Fig. 7 but with bias computed as $b_{\mathrm{hh}}^{2}(r)=\xi_{\mathrm{hh}}(r) / \xi_{\mathrm{mm}}(r)$.

above $2 \times 10^{13} h^{-1} \mathrm{M}_{\odot}$ for the three cosmological model here considered at redshifts $z=0, z=0.5$ and $z=1$. On small scales we find that the halo correlation function quickly drops as a consequence of the halo exclusion. We find that the scale where this phenomenon takes place is the same for all the cosmological models.

For each realization of the simulations $\mathrm{H} 0, \mathrm{H} 3$ and $\mathrm{H} 6$ we compute the bias using the two definitions mentioned at the beginning of this subsection: $b_{\mathrm{hm}}(r)=\xi_{\mathrm{hm}}(r) / \xi_{\mathrm{mm}}(r)$ and $b_{\mathrm{hh}}(r)=$ $\sqrt{\xi_{\mathrm{hh}}(r) / \xi_{\mathrm{mm}}(r)}$. In Fig. 7 we show the mean value of the bias, computed as $b_{\mathrm{hm}}(r)$, and its standard deviation for each cosmological model: $\Sigma_{i} m_{\nu_{i}}=0.0 \mathrm{eV}$ (red), $\Sigma_{i} m_{\nu_{i}}=0.3 \mathrm{eV}$ (green) and $\Sigma_{i} m_{\nu_{i}}=$ $0.6 \mathrm{eV}$ (blue). The results are shown at redshifts $z=0, z=0.5$ and $z=1$. Again, we do not show the results at $z=2$ because the number of haloes at this redshift is very small, and results are very noisy. The transition from the 2-halo term and the 1-halo term is clearly visible on small scales. On large scales, we find that the bias flattens out, approaching a constant value. However, as in the above subsection, we find a weak scale-dependence of bias on large scales for the cosmological models with massive neutrinos. With horizontal lines we show the value of the bias on large scales obtained by using the Tinker fitting formula along the cold dark matter prescription (solid lines) and the matter prescription (dashed lines) for cosmologies with massive neutrinos. We find that the Tinker fitting formula plus the cold dark matter prescription reproduces reasonably well our results. As we found in the power spectrum subsection, the disagreement is larger when the matter prescription is used.

In Fig. 8 we show the results in terms of the other bias estimator: $b_{\mathrm{hh}}^{2}(r)=\xi_{\mathrm{hh}}(r) / \xi_{\mathrm{mm}}(r)$. Again, we find the Tinker prediction to work well with the cold dark matter prescription. The bias scale-dependence is also present in the cosmologies with massive neutrinos when using this definition for the bias. On small scales, the correlation function drops quickly for radii smaller than $r \sim 2,3$ $h^{-1} \mathrm{Mpc}$. This is due to the finite size of the dark matter haloes, i.e. the halo exclusion effect. 

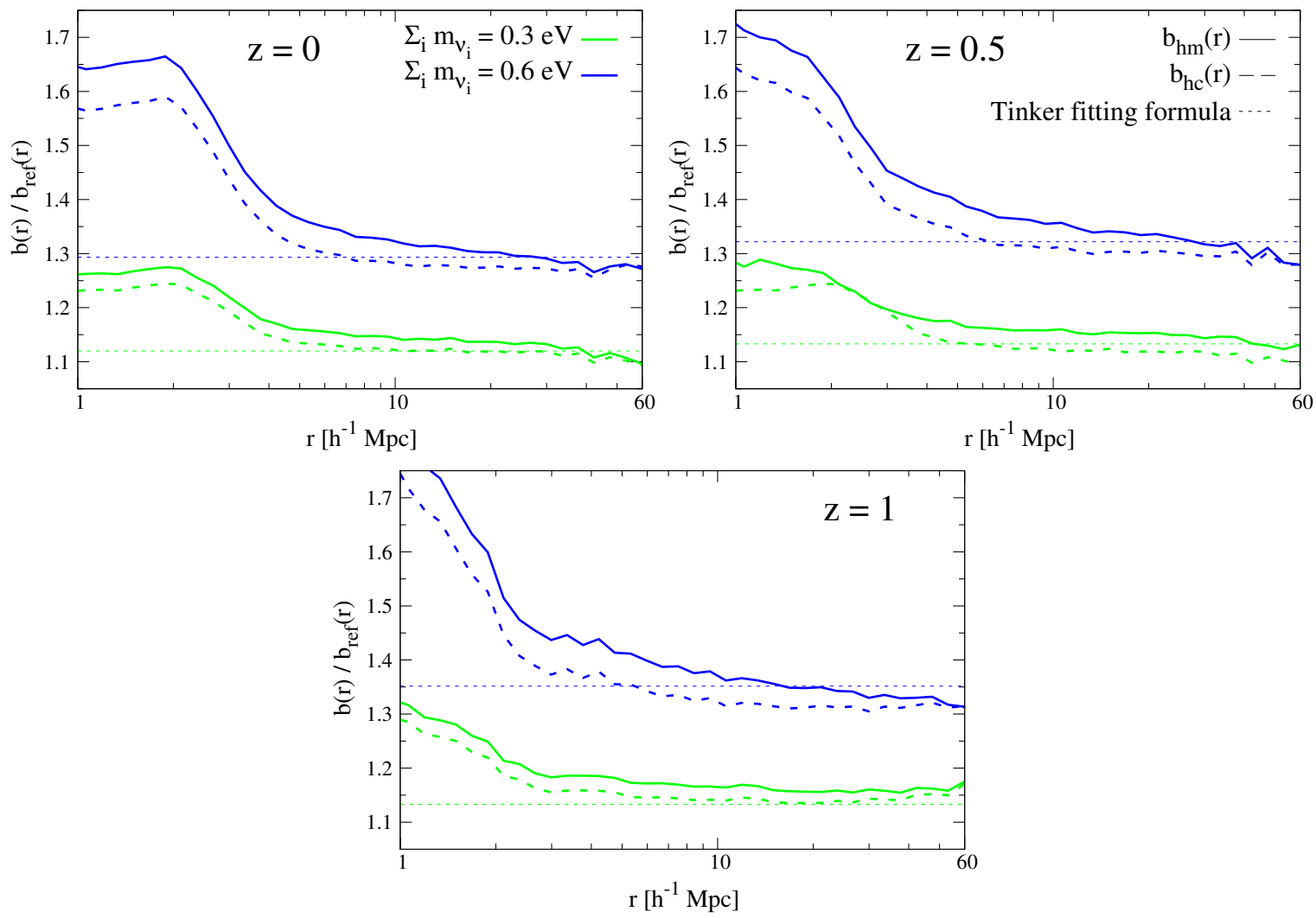

Figure 9. With solid lines we show the bias, computed as ratio of the haloes-matter cross-correlation function to the matter 2-pt autocorrelation function, for the models with $\Sigma_{i} m_{\nu_{i}}=0.3 \mathrm{eV}$ (green lines) and $\Sigma_{i} m_{\nu_{i}}=$ $0.6 \mathrm{eV}$ (blue lines) normalized by the bias of the model with massless neutrinos. The dashed lines represent the results when the bias is computed with respect to the CDM distribution, i.e. $b_{\mathrm{hc}}(r)=\xi_{\mathrm{hc}}(r) / \xi_{\mathrm{cdm}}(r)$. The prediction of the Tinker fitting formula, using the cold dark matter prescription for massive neutrinos, is shown with dotted lines.

We now check whether the scale-dependent bias we find in the simulations with massive neutrinos is suppressed if the bias is computed with respect to the spatial distribution of the CDM, as we found in the above subsection. In Fig. 9 we plot with solid lines the bias, computed with respect to the total matter distribution $b_{\mathrm{hm}}(r)$, for the models with $\Sigma_{i} m_{\nu_{i}}=0.3 \mathrm{eV}$ (green) and $\Sigma_{i} m_{\nu_{i}}=0.6 \mathrm{eV}$ (blue), normalized by bias of the cosmological model with $\Sigma_{i} m_{\nu_{i}}=0.0 \mathrm{eV}$. The scale-dependent bias is evident on large scales, in particular for the model with $\Sigma_{i} m_{\nu_{i}}=0.6 \mathrm{eV}$ and at $z=1$. With dashed lines we show the results of computing the bias with respect to the CDM distribution, i.e. using $b_{\mathrm{hm}}(r)=\xi_{\mathrm{hc}}(r) / \xi_{\mathrm{cdm}}(r)$, with $\xi_{\mathrm{cdm}}(r)$ being the correlation function of the CDM component. On large scales, the scale-dependence is highly reduced, resulting in a ratio to the massless case almost flat. We also show the values of the bias ratio on large scales obtained by using the Tinker fitting formula along with the cold dark matter prescription for massive neutrinos. Even though the agreement with our results is not perfect, in particular for the model with $\Sigma_{i} m_{\nu_{i}}=0.6 \mathrm{eV}$, it is much better than the one we get by using the Tinker fitting formula with the matter prescription.

\section{Clustering of galaxies}

In this section we populate the haloes of the different cosmological models with a realistic population of galaxies to study: 1) whether the distribution of galaxies in cosmological models with massive neutrinos can reproduce the observed galaxy clustering; and 2) to understand to what extent the 
distribution of galaxies within dark matter haloes, that is on Mpc scales, is affected by massive neutrinos. We address these issues in the framework of halo occupation distribution (HOD) models that we have adopted to build mock galaxy catalogues. These investigations should be regarded as a first step towards the more ambitious goal of constraining neutrino masses from small scales galaxy clustering. This section is split into 2 subsections. In the subsection 4.1 we describe the method we use to construct mock galaxy catalogues for a particular cosmological model, whereas in the subsection 4.2 we investigate the impact of neutrino masses into the galaxy clustering properties.

\subsection{Construction of mock galaxy catalogues}

In this subsection we describe the procedure we use to construct mock galaxy catalogues by populating with galaxies the dark matter haloes of a particular cosmology using a simple HOD model.

The HOD is a framework used to link the distribution of galaxies to that of dark matter haloes [59-65]. Within this framework, the galaxy bias is completely specified by two ingredients: 1) the probability, $P(N \mid M)$, that a halo of mass $M$ hosts $N$ galaxies of a given type; 2 ) the relation between the velocity and spatial distribution of the dark matter and the galaxies within haloes.

We now describe the method we use to construct mock galaxy catalogues from the snapshots of our N-body simulations. For a given snapshot of an N-body simulation, we first identify all the dark matter haloes and extract their properties using the SUBFIND algorithm [47] (see section 2 for details). The virial radius of each halo is defined as the radius at which the mean density within it is 200 times the mean density of the universe. In this work we focus on the clustering properties of galaxies at low redshift, and thus, we only use the $z=0 \mathrm{~N}$-body snapshots.

To populate the dark matter haloes with mock galaxies we follow the scheme of $[64,65]$, which consists of the following steps: 1) haloes with masses larger than $M_{\min }$ contain one central galaxy: haloes with masses smaller than $M_{\text {min }}$ do not host any, central or satellite, galaxy. Thus, only haloes hosting a central galaxy can host satellite galaxies. 2) the number of satellite galaxies within haloes of mass $M$ follows a Poisson distribution with a mean given by the power-law $\langle\mathrm{N}\rangle_{M}=\left(M / M_{1}\right)^{\alpha}$. We place the central galaxy at the center of the dark matter halo, and we assume that the distribution of satellites follows that of the underlying cold dark matter. We achieve this by setting the satellite galaxies on top of cold dark matter particles randomly selected within the virial radius of the halo.

Thus, our HOD model has three free parameters: $M_{\min }, M_{1}$ and $\alpha$. Our aim here is to find, for each cosmological model, the values of those parameters by requiring two conditions: 1) that the galaxy mock catalogue reproduces the observational galaxy clustering measurements; 2) that the number density of the galaxies in the mock matches the observations. As we will see below, the HOD parameter values depend on the type of galaxies used to populate the haloes. We have chosen to populate the dark matter haloes with galaxies with $M_{r}-5 \log _{10} h<-21$ and $M_{r}-5 \log _{10} h<-21.5$ (where the magnitudes are in the ${ }^{0.1} r$ band), to directly compare our results with the small scale galaxy clustering data from the main galaxy sample of SDSS-II DR7 [66].

One of the conditions we require when constructing the mock galaxy catalogues is that their galaxy number density equals the one obtained from the observations, in particular, from the luminosity function. We emphasize that we do not attempt to create mock galaxy catalogues that reproduce both the galaxy clustering measurements and the luminosity function of the galaxies. For the observational luminosity function we use the Schechter luminosity function with the parameter values presented in Blanton et al. ${ }^{8}$ [67]. Thus, the mean galaxy number density of the mocks containing galaxies brighter than -21 is equal to $1.11 \times 10^{-3} \mathrm{~h}^{3} \mathrm{Mpc}^{-3}$, whereas this number shrinks to $2.85 \times 10^{-4} h^{3} \mathrm{Mpc}^{-3}$ for the mocks containing galaxies with magnitudes smaller than -21.5.

Chosen a galaxy type, the values of the parameters $M_{1}$ and $\alpha$ are sampled from the $M_{1}-\alpha$ plane, whereas the value of $M_{\min }$ is chosen by requiring that the galaxy number density of the mock equals the one obtained from the luminosity function. Once the values of all the HOD parameters are selected, we create a mock galaxy catalogue by following the procedure described above, and compute its 2-pt auto-correlation function $\xi(r)$. Next, we calculate the projected correlation function of the mock galaxy catalogue

\footnotetext{
${ }^{8}$ The value of the parameters are: $\phi_{*}=1.49 \times 10^{-2} h^{3} \mathrm{Mpc}^{-3}, M_{*}-5 \log _{10} h=-20.44$ and $\alpha=-1.05$. They have been taken from [67]
} 


$$
w_{p}\left(r_{p}\right)=\int_{r_{p}}^{\infty} d r \frac{2 r \xi(r)}{\sqrt{r^{2}-r_{p}^{2}}},
$$

and compare it with the observational results from [66]. The comparison is quantified using the standard $\chi^{2}$ statistics

$$
\chi^{2}=\sum_{i, j}\left[w_{p}\left(r_{p_{i}}\right)-\tilde{w}_{p}\left(r_{p_{i}}\right)\right] C_{i j}^{-1}\left[w_{p}\left(r_{p_{j}}\right)-\tilde{w}_{p}\left(r_{p_{j}}\right)\right]
$$

where $\tilde{w}_{p}\left(r_{p_{i}}\right)$ and $C_{i j}$ are the observational projected correlation function and its covariance matrix ${ }^{9}$. The $M_{\min }-\alpha$ parameter space plane is scanned until the $\chi^{2}$ minimum is found. The method used to find the minimum is the following: we first compute the value of the $\chi^{2}$ on each of the points of a coarse-grid where the parameter values are allowed to vary within a wide interval. Then, we make the grid finer over the interval in which the smallest values of $\chi^{2}$ lie. We keep making the grid finer and finer until the $\chi^{2}$ minimum is stable.

We note that we are not taking into account the errors associated to the measurement of the projected correlation function of mock galaxies. As a consequence, the values of the $\chi^{2}$ for two mock galaxy catalogues created with the same values of the HOD parameters can be different. This is because by changing the random seed we may, for instance, populate more the outer regions of the dark matter haloes, and this will affect both the shape and the amplitude of the projected mock galaxy correlation function.

The 2-pt autocorrelation function of the mock galaxy catalogues is computed in real-space using the Landy-Szalay estimator [57], in the same way as explained in section 3.2. The random catalogue is constructed by filling the simulation box with four hundred thousand particles, randomly distributed within the interior volume. The number of points in the random catalogue is between two and ten times the number of galaxies in our mock catalogues. We have explicitly checked that for radii larger than $\sim 0.3 h^{-1} \mathrm{Mpc}$ our results, in terms of the autocorrelation function, do not change if we increase the number of points in the random catalogue. However, at radii smaller than $\sim 0.3 h^{-1} \mathrm{Mpc}$ our results are sensitive to the number of random points and to the binning used to compute the autocorrelation function. Moreover, on those small scales the SDSS galaxy clustering data may be affected by systematic errors arising from fiber collision. Therefore, we choose not to include the points with $r_{p}<0.3 h^{-1} \mathrm{Mpc}$ in our analysis ${ }^{10}$. We have also excluded from our analysis the noisy bins at $r_{p}>40 h^{-1} \mathrm{Mpc}$. The 2-pt autocorrelation function has been computed in 60 logarithmically equally spaced bins between $r=0.1 h^{-1} \mathrm{Mpc}$ and $r=75 h^{-1} \mathrm{Mpc}$. We have checked that our results are converged against the number of intervals and the maximum radius used to count pairs ${ }^{11}$.

We create mock galaxy catalogues, using the procedure described above, for cosmologies with massless and massive neutrinos to investigate how well our mock galaxy catalogues can reproduce the SDSS small scale galaxy clustering measurements, and to studying how galaxies should populate the dark matter haloes in the different cosmological models. As an example, we show in Fig. 10 the results of populating the dark matter haloes, at $z=0$, of the simulation L0-Planck with galaxies with magnitudes $M_{r}-5 \log _{10} h<-21$. The values of the cosmological parameters of the simulation, together with the values of the HOD parameters that better reproduce the measured projected correlation function are shown in the upper-right panel. The upper-left panel shows the value of the parameter $M_{\min }$ as a function of $M_{1}$ and $\alpha$. We find that the function $M_{\min }=M_{\min }\left(M_{1}, \alpha\right)$ displays a maximum when moving along lines with $M_{1}$ constant. In order to understand that feature, we should take into account two competing effects. The mean number density of satellite galaxies is given by:

$$
\langle N\rangle_{\text {satellites }}=\int_{M_{\min }}^{\infty}\left(\frac{M}{M_{1}}\right)^{\alpha} n(M, z) d M
$$

\footnotetext{
${ }^{9}$ The covariance matrices have been kindly provided by Idit Zehavi (private communication).

${ }^{10}$ Due to computational limitations, we are unable to perform the analysis described here with a random catalogue containing a number of points much larger than 400000.

${ }^{11}$ Our tests are performed over the projected correlation function.
} 


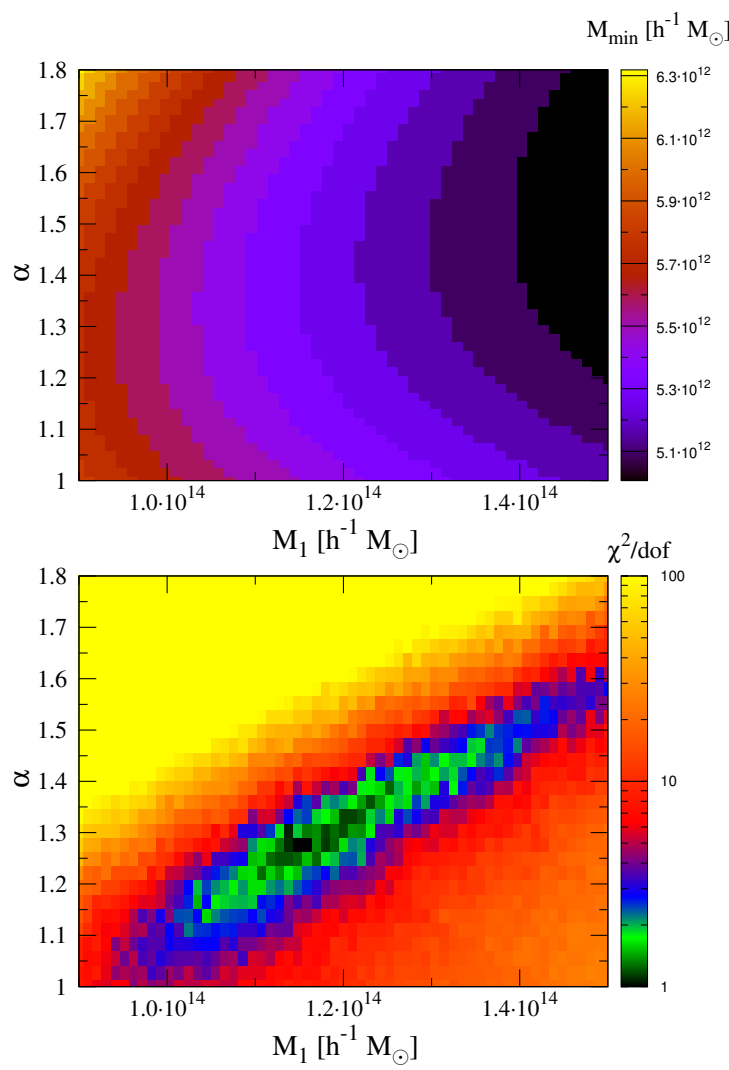

$$
\begin{array}{r}
512^{3} \text { CDM particles }: 500 \mathrm{~h}^{-1} \mathrm{Mpc} \\
\Omega_{\mathrm{CDM}}=0.2685: \Omega_{\mathrm{b}}=0.049 \\
\Omega_{\Lambda}=0.6825: \Omega_{\mathrm{v}}=0.00 \\
\sigma_{8}=0.834: \mathrm{h}=0.6711
\end{array}
$$

$$
\begin{gathered}
\mathrm{M}_{1}=1.15 \times 10^{14} \mathrm{~h}^{-1} \mathrm{M}_{\odot}: \alpha=1.27 \\
\mathrm{M}_{\min }=5.33 \times 10^{12} \mathrm{~h}^{-1} \mathrm{M}_{\odot}
\end{gathered}
$$

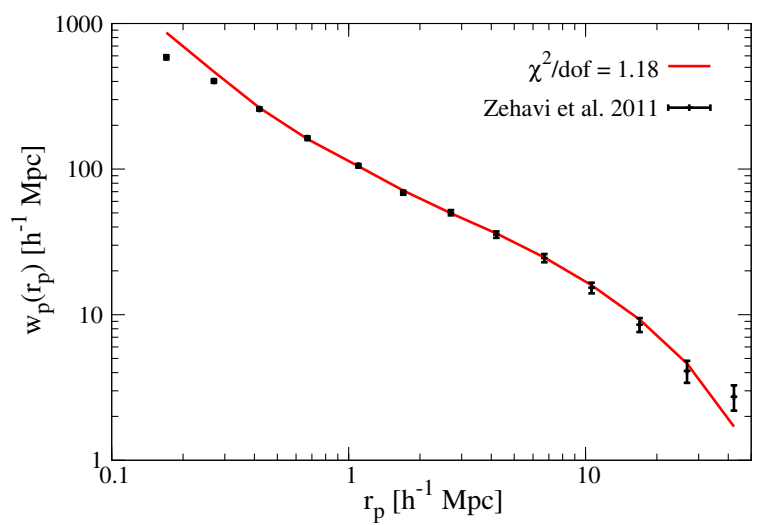

Figure 10. An example of the HOD analysis, performed in this case on top of the L0-Planck simulation. The cosmological parameters of that simulation are reported in the upper-right panel. We construct mock galaxy catalogues, for galaxies with $M_{r}-5 \log _{10} h<-21$, requiring that their number density to be equal to this obtained from the luminosity function of Blanton et al. [67]. The value of the parameter $M_{\min }$ is fixed by demanding the former condition. In the upper-left panel we show the value of $M_{\min }$ as a function of the parameters $\alpha$ and $M_{1}$. For each point in the HOD parameter space we create a mock galaxy catalogue and compute its projected correlation function, comparing it with observations by using the $\chi^{2}$ statistics. In the bottom-left panel we plot the value of $\chi^{2}$, normalized to the number of degrees of freedom, as function of $\alpha$ and $M_{1}$. The values of the HOD parameters that better reproduce observations for this cosmology are shown at the bottom of the upper-right panel. The projected correlation function for those HOD parameters is shown in the bottom-left panel, together with the observational measurements. Note that when comparing with observations we only use the $w_{p}$ points with $r>0.3 h^{-1} \mathrm{Mpc}$ and $r<30 h^{-1} \mathrm{Mpc}$.

where $n(M, z) d M$ is the number density of haloes with masses between $M$ and $M+d M$. Whereas the number of satellite galaxies residing in haloes with masses smaller than $M_{1}$ decrease with increasing $\alpha$, the number of satellites galaxies within haloes of masses larger than $M_{1}$ increases with $\alpha$. For low values of $\alpha$, the total number of satellites is dominated by those living in haloes with masses higher than $M_{1}$, and therefore, as $\alpha$ increases, the total number of satellites increases. This implies that the number of central galaxies should shrink (to keep the same galaxy number density), i.e. the value of $M_{\min }$ should increase with $\alpha$. If the value of $\alpha$ is large, the satellite population will be dominated by galaxies residing in haloes with masses smaller than $M_{1}$. In this situation, a larger value of $\alpha$ will further shrink the number of satellites. Therefore, the number of central galaxies should increase, which implies that the value for $M_{\min }$ should decrease with increasing $\alpha$.

On the bottom-left panel we plot the value of the $\chi^{2}$, normalized to the number of degrees of freedom, $\chi^{2} /$ d.o.f., as a function of the values of the HOD parameters. As stated above, the comparison between the clustering of our mock galaxy catalogues and the SDSS data is performed for 


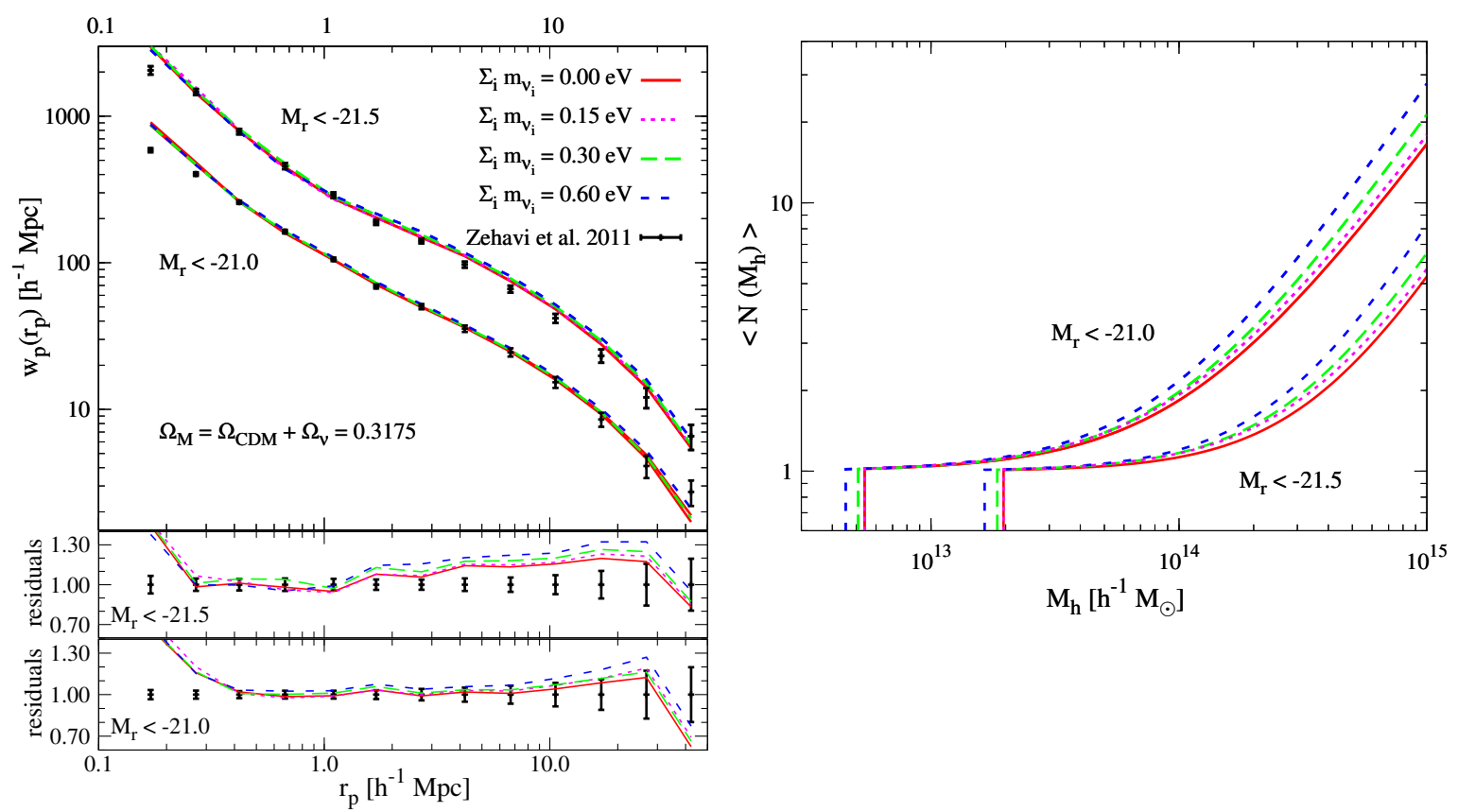

Figure 11. In the upper-right panel we show with black points the $w_{p}$ measurements for galaxies with $M_{r}-5 \log _{10} h<-21$ (bottom points) and -21.5 (top points) from Zehavi et al. [66]. The error bars represent the diagonal terms of the covariance matrix. The projected correlation function of the mock galaxy catalogues created with HOD parameter values that better reproduce those observations are shown for three different cosmologies with different sums of the neutrino masses: $0 \mathrm{eV}$ (solid red), $0.3 \mathrm{eV}$ (long dashed green) and $0.6 \mathrm{eV}$ (short dashed blue). For clarity, the observational measurements and our results for the galaxies with magnitudes brighter than -21.5 have been displaced vertically by a factor of 2 . The relative differences between those and observations are show in the middle panel (for galaxies with $M_{r}-5 \log _{10} h<-21.5$ ) and bottom panel (for galaxies with $M_{r}-5 \log _{10} h<-21$ ). The right panel shows the mean number of galaxies as a function of the mass of the dark matter halo hosting them for the three different cosmologies.

points in the projected correlation function with radii larger than $0.3 h^{-1} \mathrm{Mpc}$ and smaller than 40 $h^{-1} \mathrm{Mpc}$ by using the full covariance matrix. We find a strong correlation between the parameters $\alpha$ and $M_{1}$. We note that because we are not taking into account the errors in the measurement of the mock galaxy correlation function, the values of the $\chi^{2}$ not only depend on the HOD parameters but also on the mock catalog realization, i.e. on the set of random numbers we use to populate the dark matter haloes with galaxies. This is clearly reflected in the $\chi^{2}=\chi^{2}\left(\alpha, M_{1}\right)$ plot: the $\chi^{2}$ function is not a smooth function of the parameters $\alpha$ and $M_{1}$, but the scatter induced by the particular realization used to create the mock galaxy catalogue appears as bins with colors slightly displaced to the color they would have if the mean of several realizations would be use.

Finally, the projected correlation function of the mock galaxy catalogue constructed with values of the HOD parameters that minimize the $\chi^{2}$ is plotted on the bottom-right panel. The black points represent the observational measurements, taken from Zehavi et al. [66], where the error bars correspond to the square root of the covariance matrix diagonal terms. We find that with this simple HOD model we can reproduce pretty well the projected correlation function for galaxies with magnitudes brighter than -21 , obtaining a minimum value of $\chi^{2} /$ d.o.f. equal to 1.18 . We quote the values of the HOD parameters that minimize the $\chi^{2}$ on table 2 .

\subsection{HOD: galaxy clustering properties}

In the above section we have explained the method we use to construct the mock galaxy catalogues for a particular cosmological model. In this subsection we investigate how well our mock galaxy 


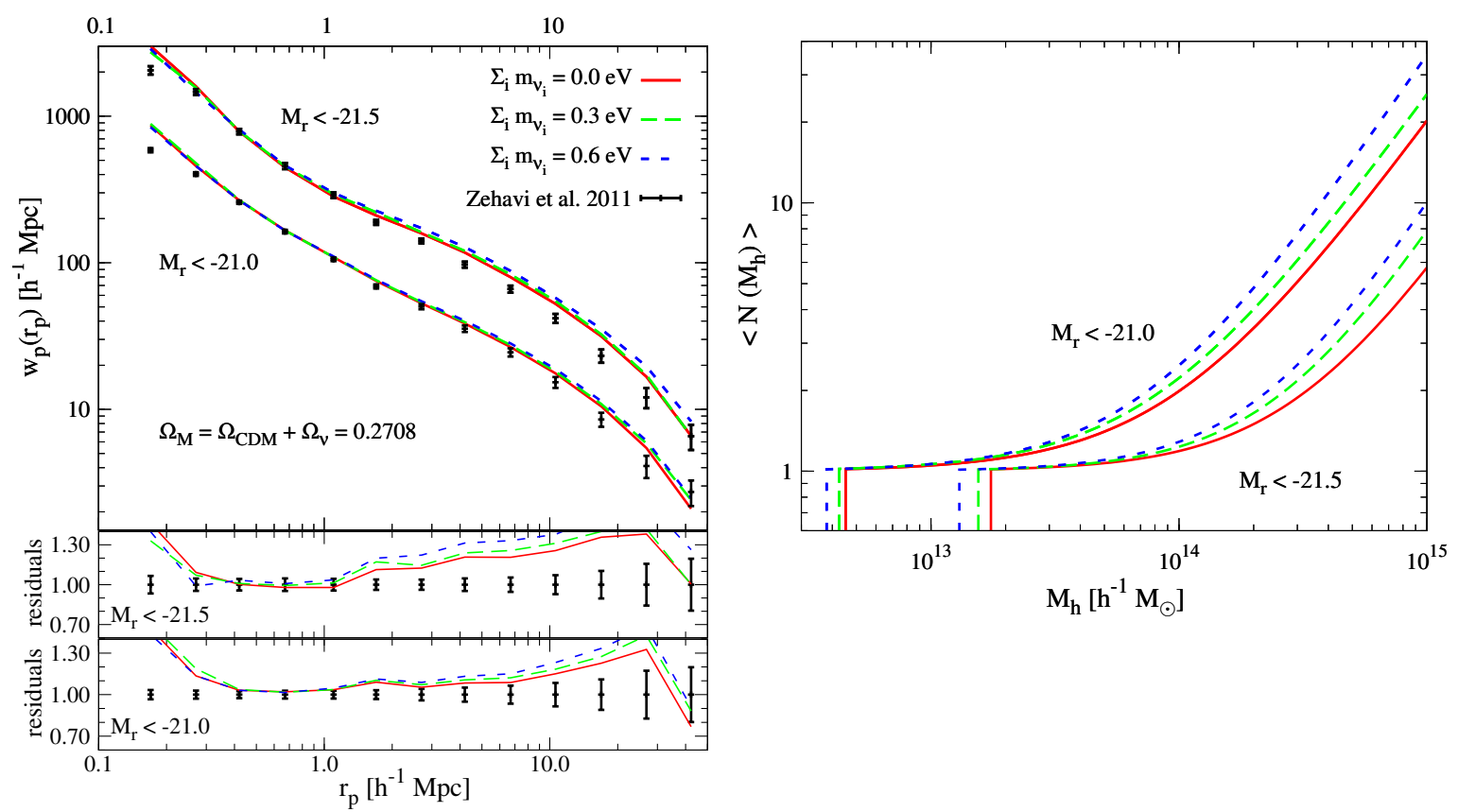

Figure 12. Same as Fig. 11 but with cosmological models with $\Omega_{\mathrm{m}}=0.2708$ instead of $\Omega_{\mathrm{m}}=0.3175$.

catalogues can reproduce the observational clustering measurements, and how the distribution of galaxies within dark matter haloes is impacted by the masses of neutrinos.

We first investigate how well we can reproduce the observed projected correlation function for cosmologies with massive neutrinos. We construct mock galaxy catalogues, containing galaxies with magnitudes brighter than -21 , for those cosmological models by repeating the above procedure on top of the simulations with $\Sigma_{i} m_{\nu_{i}}=0.15 \mathrm{eV}$ (L15-Planck), $\Sigma_{i} m_{\nu_{i}}=0.30 \mathrm{eV}$ (L3-Planck) and $\Sigma_{i} m_{\nu_{i}}=0.60$ $\mathrm{eV}$ (L6-Planck). We note that those simulations share the value of the parameters $\Omega_{\mathrm{cdm}}$ and $A_{s}$ with the simulation with massless neutrinos used above (L0-Planck). The projected correlation functions of the mock galaxy catalogues created with the HOD parameters that better reproduce the clustering observations are shown in the upper-left panel of Fig. 11. In the bottom-left panel of that figure we show the relative difference between observations and our mock catalogues. We find that the models with massive neutrino can also reproduce pretty well the observational measurements: $\chi^{2} /$ d.o.f. $=$ $1.28,1.33$ and 1.35 for the cosmological models with neutrino masses equal to $\Sigma_{i} m_{\nu_{i}}=0.15,0.30$ and $0.60 \mathrm{eV}$, respectively. However, we find that HOD models over-predict galaxy correlation at large separations, where the 2-halo term dominates.

We repeat the whole analysis, for the same above cosmological models, by creating mock galaxy catalogues containing galaxies with magnitudes $M_{r}-5 \log _{10} h<-21.5$. The results are also shown in Fig. 11. For this case, we find that the the galaxy mocks catalogues do not reproduce that well the observations: $\chi^{2} /$ d.o.f. $=2.25,2.73,3.25$ and 4.88 for the models with $\Sigma_{i} m_{\nu_{i}}=0.00,0.15,0.30$ and $0.60 \mathrm{eV}$ respectively. As for the mocks with galaxies brighter than -21 , we find that the disagreement between our results and observations increases with the neutrino masses. The disagreement arises primarily on large scales, where the 2-halo term dominates. The right panel of Fig. 11 shows the average number of galaxies hosted by dark matter haloes as a function of the halo mass, obtained directly from the values of the HOD parameters that best reproduce the observations. For galaxies with luminosities above a certain threshold, we find that the most massive haloes should contain more galaxies in cosmologies with massive neutrinos than in cosmologies with massless neutrinos. Moreover, those galaxies can be hosted by haloes of lower mass for the massive neutrino cosmologies.

Now we focus our analysis into the impact of the cosmological parameter $\Omega_{\mathrm{m}}$ on the clustering 
properties of our mock galaxy catalogues. We have constructed mock galaxy catalogues using the $z=0$ snapshots of the simulations L0, L3 and L6, which basically differ from the previous ones in the value of $\Omega_{\mathrm{m}}$ (see table 1). The results are shown in Fig. 12. Using a higher value for $\Omega_{\mathrm{m}}$ further increases the mismatch between HOD model and observations that, as in the previous tests, increases with scale. For the mocks containing galaxies with $M_{r}-5 \log _{10} h<-21$ the values of the minimum $\chi^{2} /$ d.o.f. are $1.85,2.39$ and 2.74 for the cosmologies with $\Sigma_{i} m_{\nu_{i}}=0.0,0.3$ and $0.6 \mathrm{eV}$ respectively. The disagreement is even worst for the mock catalogues hosting galaxies with $M_{r}-5 \log _{10} h<-21.5$ : $\chi^{2} /$ d.o.f. $=3.88,5.44$ and 8.13 for the models with $\Sigma_{i} m_{\nu_{i}}=0.0,0.3$ and $0.6 \mathrm{eV}$ respectively. Again, we find that the disagreement between our mock catalogues and the observational data increases with the neutrino masses, arising mainly on large scales. For each cosmological model and for each mock galaxy catalogue we list on table 2 the values of the HOD parameters that minimize the $\chi^{2} /$ d.o.f. together with the value of it.

Next, we study which is the influence of the parameter $\Omega_{\nu}$ on the clustering properties of our mock catalogues. In particular, we would like to know if the main effect in the galaxy clustering properties arise from $\Omega_{\nu}$, or whether it is mainly related to the values of other cosmological parameters such as $\Omega_{\mathrm{cdm}}$ or $\sigma_{8}$. To answer this question we have created mock galaxy catalogues using the simulations L6-Planck, L6, L6-1 and L6-2. All those simulations represent cosmological models with neutrinos of masses $\Sigma_{i} m_{\nu_{i}}=0.60 \mathrm{eV}$. The differences among them are in the value of the cosmological parameters $\Omega_{\mathrm{m}}$ and $\sigma_{8}$. The value of the couple $\left(\Omega_{\mathrm{m}}, \sigma_{8}\right)$ is $(0.3175,0.69),(0.2708,0.675),(0.2708,0.832)$ and $(0.30,0.749)$ for the simulations L6-Planck, L6, L6-1 and L6-2 respectively. The projected correlation function of the HOD galaxies that best fits observations is shown in Fig. 13, and the HOD parameters together the value of the $\chi^{2} /$ d.o.f. are listed in table 2 . We find that the simulations in which our HOD model reproduces better the observed galaxy clustering are L6-Planck and L6-2. Those simulations have values of the parameter $\Omega_{\mathrm{cdm}}$ higher than the simulations L6 and L6-1. The impact of the parameter $\sigma_{8}$ seems to be less important. The simulation L6-1 has a value of $\sigma_{8}=0.832$ significantly larger than all the other simulations, but we find that values of the $\chi^{2} /$ d.o.f. equal to 3.81 and 6.71 for galaxies with magnitudes brighter than -21 and -21.5 respectively, in comparison with the simulations L6-Planck and L6-2, for which we find $\chi^{2} /$ d.o.f. $=1.35$ and 4.88 (for L6-Planck) and $\chi^{2} /$ d.o.f. $=$ 1.32 and 3.42 (for L6-2). However, the value of $\sigma_{8}$ may be the reason why our mock galaxy catalogue reproduces better the measurements for galaxies with magnitudes smaller than -21.5 in the case of the simulation L6-2 than in the simulation L6-Planck. Overall, we find that our mock galaxy catalogues reproduce pretty well the projected correlation function on small scales (the 1-halo term) for all the cosmological models.

It can be argued that the values of the parameters $\Omega_{\Lambda}, h$ or $n_{s}$ also play an important role on our results. However since the models that best fit the data, all models with the suffix -Planck plus model L6-2, use a similar $\Omega_{\mathrm{cdm}}$ while the other parameters span a wide range of values suggests that galaxy clustering is mostly sensitive to the value of the cosmological parameter $\Omega_{\mathrm{cdm}}$.

We have checked the robustness of our results by running a N-body simulation with the same mass resolution as the previous simulations but with a box size twice as large. This simulation, labeled as H0-Planck-LV on table 1 has the same cosmological parameters as the simulation L0Planck, although the former has a box size equal to $1000 h^{-1} \mathrm{Mpc}$ whereas the size of the latter is of $500 h^{-1} \mathrm{Mpc}$. The resolution in both simulations is the same since H0-Planck-LV contains $1024^{3}$ CDM particles (to compare with the $512^{3}$ of L0-Planck). We have computed the values of the HOD parameters for which the mock galaxy catalogues best reproduce the projected correlation function of galaxies with $M_{r}-5 \log _{10} h<-21.5^{12}$. We find that the best fit HOD parameters are slightly different to those obtained for the simulation L0-Planck. However, the minimum value of $\chi^{2} /$ d.o.f. remains the same, pointing out that the discrepancies arise from the 2-halo term, which, in our simple HOD implementation, is mainly determined by the cosmological model. We emphasize that the purpose of this work is not to determine the exact values of HOD parameters that reproduce the observations, but to study the impact of massive neutrinos on the galaxy clustering properties. Thus, our main aim here is to investigate the relative differences among the different models.

\footnotetext{
${ }^{12}$ Note that for galaxies with $M_{r}-5 \log _{10} h<-21$ the mock catalogues would contain more than 1 million of galaxies, making the analysis presented here too expensive from the computational point of view.
} 


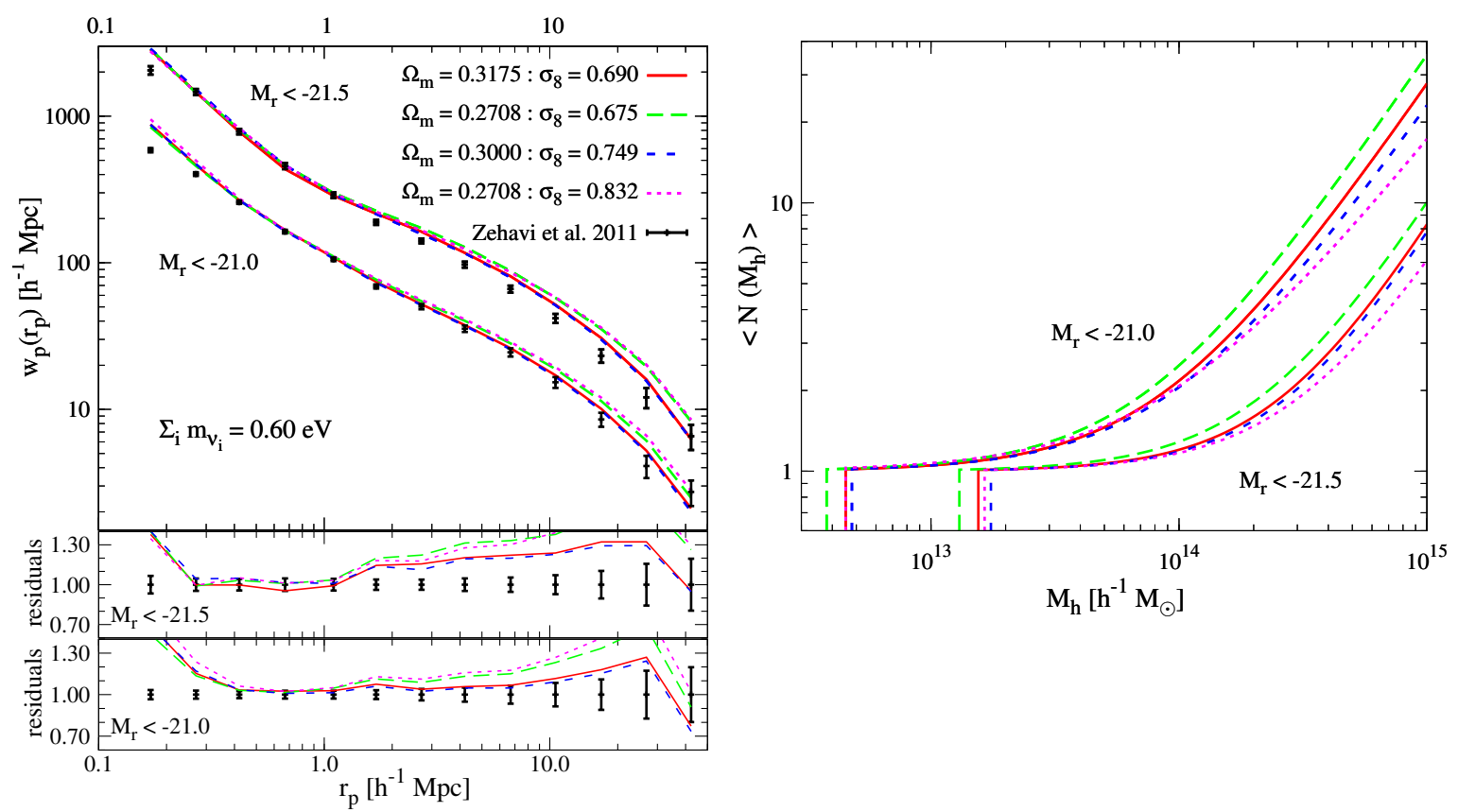

Figure 13. Same as Fig. 11 but with cosmological models with neutrinos with masses equal to $\Sigma_{i} m_{\nu_{i}}=$ $0.6 \mathrm{eV}$. The mock galaxy catalogues have been constructed over the simulations L6-Planck (red), L6 (green), L6-2 (blue) and L6-1 (magenta). The values of the parameters $\Omega_{\mathrm{m}}$ and $\sigma_{8}$ are shown on the top-right corner of the upper-left panel for the different cosmological models.

The pretty large disagreement between the projected correlation function of our $M_{r}-5 \log _{10} h<$ -21.5 mock galaxy catalogues and the observed one may be due to the simplistic HOD model we have used to populate the dark matter haloes. Zehavi et al. 2004 [68] found that the projected correlation function of the brightest galaxies is better reproduced if a different HOD model is used. The large values of the $\chi^{2}$ may also be due to an underestimation of the covariance matrix, as claimed also by [66].

Our results suggest that with the simple HOD modeling we have used in this paper we can construct mock galaxy catalogues that reproduce the SDSS small scale galaxy clustering data, for radii smaller than $\sim 1.5 h^{-1} \mathrm{Mpc}$, for all the cosmological models, independently of the neutrino masses or the values of $\Omega_{\mathrm{m}}$ and $\sigma_{8}$. However, we find that for radii larger than $\sim 2 h^{-1} \mathrm{Mpc}$, the shape and amplitude of the projected correlation function of our mock catalogues is mainly determined by the value of the cosmological parameter $\Omega_{\mathrm{cdm}}$. Since the cosmological models we have used in this paper share the value of the parameter $\Omega_{\mathrm{m}}=\Omega_{\mathrm{cdm}}+\Omega_{\nu}$, we find that the largest the neutrino masses the largest the disagreement on large scales, but this is just a consequence of the different value of the parameter $\Omega_{\mathrm{cdm}}$ among the models.

Finally, we quantify the dependence of the values of the HOD parameters with the neutrino masses. Table 2 shows the values of the HOD parameters, for each mock catalogue we have created, that better reproduce the SDSS galaxy clustering measurements. We find that the parameter $\alpha$ exhibits a very weak dependence on the masses of the neutrinos and with cosmology. However, when comparing models with the same values of the parameters $\Omega_{\mathrm{m}}$ and $A_{s}$, we find that the general trend indicates that this parameters increases with $\Sigma_{i} m_{\nu_{i}}$. On the other hand, the values of the parameters $M_{1}$ and $M_{\min }$ are strongly dependent on the neutrino masses. By comparing the values of those parameters among cosmological models that share the values of $\Omega_{\mathrm{m}}$ and $A_{s}$ (L6-Planck, L3-Planck, L15-Planck and L0-Planck on one hand, and L6, L3 and L0 on the other hand) we find that a simple relation of the form $\log \left(M_{1}\right)=a+b \Sigma_{i} m_{\nu_{i}}$ and $\log \left(M_{\min }\right)=a+b \Sigma_{i} m_{\nu_{i}}$ reproduces pretty well the 


\begin{tabular}{|c|c|c|c|c|c|c|c|c|}
\hline$M_{r}-5 \log _{10} h$ & $\begin{array}{c}\text { Simulation } \\
\text { name }\end{array}$ & $\Omega_{\mathrm{m}}$ & $\begin{array}{c}\sigma_{8} \\
(\mathrm{z}=0)\end{array}$ & $\begin{array}{c}\Sigma_{i} m_{\nu_{i}} \\
(\mathrm{eV})\end{array}$ & $\begin{array}{c}M_{1} \\
\left(h^{-1} \mathrm{M}_{\odot}\right)\end{array}$ & $\begin{array}{c}M_{\min } \\
\left(h^{-1} \mathrm{M}_{\odot}\right)\end{array}$ & $\chi^{2} /$ d.o.f. \\
\hline \hline-21.0 & L0-Planck & 0.3175 & 0.834 & 0.00 & $1.15 \times 10^{14}$ & 1.27 & $5.33 \times 10^{12}$ & 1.18 \\
\hline-21.0 & L15-Planck & 0.3175 & 0.799 & 0.15 & $1.06 \times 10^{14}$ & 1.26 & $5.19 \times 10^{12}$ & 1.28 \\
\hline-21.0 & L3-Planck & 0.3175 & 0.761 & 0.30 & $1.02 \times 10^{14}$ & 1.32 & $4.91 \times 10^{12}$ & 1.33 \\
\hline-21.0 & L6-Planck & 0.3175 & 0.690 & 0.60 & $8.90 \times 10^{13}$ & 1.36 & $4.47 \times 10^{12}$ & 1.35 \\
\hline-21.0 & L0 & 0.2708 & 0.832 & 0.00 & $1.01 \times 10^{14}$ & 1.29 & $4.48 \times 10^{12}$ & 1.85 \\
\hline-21.0 & L3 & 0.2708 & 0.752 & 0.30 & $8.56 \times 10^{13}$ & 1.30 & $4.18 \times 10^{12}$ & 2.39 \\
\hline-21.0 & L6 & 0.2708 & 0.675 & 0.60 & $7.52 \times 10^{13}$ & 1.37 & $3.73 \times 10^{12}$ & 2.74 \\
\hline-21.0 & L6-1 & 0.2708 & 0.832 & 0.60 & $9.39 \times 10^{13}$ & 1.18 & $4.30 \times 10^{12}$ & 3.81 \\
\hline-21.0 & L6-2 & 0.3000 & 0.749 & 0.60 & $9.59 \times 10^{13}$ & 1.32 & $4.67 \times 10^{12}$ & 1.32 \\
\hline \hline-21.5 & L0-Planck & 0.3175 & 0.834 & 0.00 & $3.84 \times 10^{14}$ & 1.53 & $1.95 \times 10^{13}$ & 2.25 \\
\hline-21.5 & L15-Planck & 0.3175 & 0.799 & 0.15 & $3.45 \times 10^{14}$ & 1.45 & $1.88 \times 10^{13}$ & 2.73 \\
\hline-21.5 & L3-Planck & 0.3175 & 0.761 & 0.30 & $3.23 \times 10^{14}$ & 1.51 & $1.77 \times 10^{13}$ & 3.25 \\
\hline-21.5 & L6-Planck & 0.3175 & 0.690 & 0.60 & $2.79 \times 10^{14}$ & 1.56 & $1.55 \times 10^{13}$ & 4.88 \\
\hline-21.5 & L0 & 0.2708 & 0.832 & 0.00 & $3.29 \times 10^{14}$ & 1.40 & $1.66 \times 10^{13}$ & 3.88 \\
\hline-21.5 & L3 & 0.2708 & 0.752 & 0.30 & $2.69 \times 10^{14}$ & 1.47 & $1.48 \times 10^{13}$ & 5.44 \\
\hline-21.5 & L6 & 0.2708 & 0.675 & 0.60 & $2.30 \times 10^{14}$ & 1.50 & $1.28 \times 10^{13}$ & 8.13 \\
\hline-21.5 & L6-1 & 0.2708 & 0.832 & 0.60 & $3.32 \times 10^{14}$ & 1.48 & $1.58 \times 10^{13}$ & 6.71 \\
\hline-21.5 & L6-2 & 0.3000 & 0.749 & 0.60 & $2.95 \times 10^{14}$ & 1.57 & $1.65 \times 10^{13}$ & 3.42 \\
\hline
\end{tabular}

Table 2. Values of the HOD parameters, for each cosmological model and for with magnitudes than a given threshold, that best fit the observational measurements.

\begin{tabular}{|c|c|c|}
\hline$M_{r}-5 \log _{10} h$ & $\Omega_{\mathrm{m}}=0.3175$ & $\begin{array}{c}\Omega_{\mathrm{m}}=0.2708 \\
(\mathrm{~L} 0, \mathrm{~L} 3, \mathrm{~L} 6)\end{array}$ \\
\hline \hline-21.0 & $\log$-Planck, L15-Planck, L3-Planck, L6-Planck $)$ & $\log \left(M_{1}\right)=32.24-0.492 \Sigma_{i} m_{\nu_{i}}$ \\
& $\log \left(M_{\min }\right)=32.37-0.416 \Sigma_{i} m_{\nu_{i}}$ & $\log \left(M_{\min }\right)=29.14-0.305 \Sigma_{i} m_{\nu_{i}}$ \\
\hline-21.5 & $\log \left(M_{1}\right)=33.57-0.502 \Sigma_{i} m_{\nu_{i}}$ & $\log \left(M_{1}\right)=33.42-0.597 \Sigma_{i} m_{\nu_{i}}$ \\
& $\log \left(M_{\min }\right)=30.61-0.391 \Sigma_{i} m_{\nu_{i}}$ & $\log \left(M_{\min }\right)=30.45-0.433 \Sigma_{i} m_{\nu_{i}}$ \\
\hline
\end{tabular}

Table 3. Dependence of the HOD parameters $M_{1}$ and $M_{\min }$ with the masses of the neutrinos for cosmological models with $\Omega_{\mathrm{m}}=0.3175$ (simulations L0-Planck, L15-Planck, L3-Planck and L6-Planck) and with $\Omega_{\mathrm{m}}=$ 0.2708 (simulations L0, L3 and L6). The fits are shown for mock galaxy catalogues with galaxies with magnitudes brighter than -21 and -21.5 .

dependence of those parameters with the neutrino masses. In table 3 we show the values of the parameters $a, b, c$ and $d$ for the two different set of cosmological models and for the two types of mock galaxy catalogues.

\section{Summary and conclusions}

We have run a large suite of $80 \mathrm{~N}$-body simulations containing CDM and neutrino particles to study the impact of massive neutrinos on the spatial distribution of dark matter haloes and galaxies. Our simulations span a total of 12 different cosmological models, with box sizes between 500 and 1000 $h^{-1} \mathrm{Mpc}$. For 6 of those cosmological model we have run 8 independent realizations, in order to make our conclusions more robust.

We have investigated the bias between the spatial distribution of dark matter haloes and that of the underlying matter with two different techniques: the power spectrum and the correlation function. With both techniques, we find that on large scales and for the simulations with massless neutrinos, 
the bias becomes scale-independent, in agreement with theoretical expectations. However, in the simulations with massive neutrinos we find a weak, scale-dependent bias on large scales. This is true as long as the total matter power spectrum, including contributions from both neutrinos and $\mathrm{CDM}$, is used in the bias estimator. If, instead, one consider the CDM power spectrum, then the scale dependence becomes much weaker. In our companion paper [39], we study in detail this point, showing that the bias becomes universal only if it is computed with respect to the distribution of CDM.

We have also adressed the $\Omega_{\nu}-\sigma_{8}$ degeneracy on the halo-matter bias. We find that bias is little sensitive to $\Sigma_{i} m_{\nu_{i}}$ if the value of the parameter $\sigma_{8}$ is kept fixed. However, in the cosmologies with massive neutrinos the bias does show some scale dependence. A nearly perfect degeneracy will hold between models sharing the value of $\sigma_{8}$, if the bias is computed with respect to the CDM linear power spectrum.

For cosmologies with massive neutrinos we find that the value of the bias on large scales is reasonably well described by the fitting formula of Tinker [55], once the cold dark matter prescription for massive neutrinos is used. This prescription relies on the use of the CDM linear matter power spectrum when computing the value of $\sigma(M)$ (or equivalently $\nu=\delta_{c} / \sigma(M)$ ), instead of the total matter linear power spectrum (matter prescription), and in replacing $\rho_{\mathrm{m}}$ with $\rho_{\mathrm{cdm}}$.

By populating the dark matter haloes with realistic galaxies we have constructed mock galaxy catalogues for a large variety of cosmological models, and we have used these simulations to investigate the impact of neutrino masses on galaxy clustering properties. We have used a simple HOD model to populate the haloes with galaxies, whose magnitudes, in the ${ }^{0.1} r$ band, are smaller than -21 and -21.5 . We have constructed our mock catalogues by requiring that: 1) the number density of the galaxies in the mocks is equal to the one obtained from the luminosity function of Blanton et al. [67]; 2) the clustering properties of the galaxies in the mocks mimic, as close as possible, the small scales galaxy clustering data of the SDSS collaboration [66]. For each cosmological model (N-body simulation) we tuned the values of the HOD parameters to achieve the requirements.

Overall, our mock galaxy catalogues can reproduce very well the projected correlation function of Zehavi et al. on small scales for all the cosmological models (the so-called 1-halo term). On large scales, however, where the projected correlation function of our mocks is determined mainly by the cosmological parameter $\Omega_{\mathrm{cdm}}$ models do not match observations that well. Our mock catalogues can reproduce the observed projected correlation function of galaxies with $M_{r}-5 \log _{10} h<-21$ with a $\chi^{2} /$ d.o.f. $\sim 1.2-1.4$, almost independently of the value of the sum of the neutrino masses, when using cosmological models with $\Omega_{\mathrm{m}}=0.3175$ (simulations L6-Planck, L3-Planck and L0-Planck). The values of the $\chi^{2}$ /d.o.f. are more sensitive to the neutrino masses if a cosmology with a lower value of $\Omega_{\mathrm{m}}=0.2708$ is used: $\chi^{2} /$ d.o.f. $\sim 1.9-2.7$ for the simulations L6, L3 and L0. We also find that the value of the cosmological parameter $\sigma_{8}$, for this HOD modeling, does not play an important role for the clustering of galaxies with magnitudes brighter than -21, but with larger values of $\sigma_{8}$ we can reproduce better the clustering measurements for galaxies with magnitudes smaller than -21.5.

Our mock catalogues cannot reproduce the projected correlation function of galaxies with magnitudes $M_{r}-5 \log _{10} h<-21.5$ well as indicated by the large $\chi^{2} /$ d.o.f. values we get: from $\sim 2-5$ for the cosmological models with $\Omega_{\mathrm{m}}=0.3175$ to $\sim 3-8$ for the cosmological models with $\Omega_{\mathrm{m}}=0.2708$. We believe that this may be due to the simplistic HOD model used to populate haloes with those galaxies. In [68] authors found than a more complex HOD parametrization could better reproduce the observational measurement for the more luminous galaxies.

We emphasize that the tensions we find, in the projected correlation function, on the 2-halo term between the observational measurements and our mock galaxy catalogues can be also alleviated by introducing some scatter in the galaxy number density from the luminosity function, i.e. by relaxing the condition we require to our mocks: that the galaxy number density of our mocks has to match the galaxy number density obtained from the luminosity function. Thus, we believe that the use of a more complex HOD model $[15,69,70]$ may help to reduce the tensions between our mocks and the observational measurements.

We have compared cosmological models that share the values of $\Omega_{\mathrm{m}}$ and $\sigma_{8}$ but differ on $\Sigma_{i} m_{\nu_{i}}$. Our results indicate that the dark matter haloes in the cosmologies with the most massive neutrinos 
have to be more densely populated to match observations. Moreover, in a massive neutrino cosmology the same galaxy population is hosted in less massive haloes that in the massless neutrino cosmology. This is however just a reflection of the impact of neutrino masses on the halo mass function.

Thus, we conclude that it is unlikely that the neutrino masses can be constrained by using smallscale galaxy clustering measurements alone. However, by combining galaxy clustering data with CMB measurements, that would fix the total amount of cold dark matter, tight bounds on the neutrino masses can be set by exploiting the small scale clustering regime of galaxy survey.

\section{Acknowledgements}

We thank Idit Zehavi for having kindly provided us with the covariance matrices of the $w_{p}\left(r_{p}\right)$ measurements. We also thank Ravi Sheth for useful comments. FVN would like to thank Simeon Bird for his help with the N-body simulations run for this paper. Calculations were performed on SOM2 and SOM3 at IFIC and on the COSMOS Consortium supercomputer within the DiRAC Facility jointly funded by STFC, the Large Facilities Capital Fund of BIS and the University of Cambridge, as well as the Darwin Supercomputer of the University of Cambridge High Performance Computing Service (http:// www.hpc.cam.ac.uk/), provided by Dell Inc. using Strategic Research Infrastructure Funding from the Higher Education Funding Council for England. FVN and MV are supported by the ERC Starting Grant "cosmoIGM". MV is also supported by I.S. INFN/PD51. ES was supported in part by NSF-AST 0908241. SS is supported by a Grant-in-Aid for Young Scientists (Start-up) from the Japan Society for the Promotion of Science (JSPS) (No. 25887012).

\section{A Power spectrum corrections and errors}

The power spectrum of a distribution of points that arise from a Poisson sampling of a underlying density field is related with that of the underlying field by (see for instance [71])

$$
P^{d}(\vec{k})=P^{c}(\vec{k})+\frac{1}{\bar{n}},
$$

where $\bar{n}$ is the points number density and $P^{d}(\vec{k})$ and $P^{c}(\vec{k})$ are the power spectra of the discrete point set and of the continuos field, respectively. However, when computing the power spectrum of the CDM particles we should not subtract the $\bar{n}^{-1}$ term (we will refer to this extra contribution in the power spectrum as shot-noise). This is because we generated the initial conditions of the CDM particles on a grid, perturbing their positions around the grid points according to the gravitational potential, and this process does not correspond to a Poisson sampling of the density field.

Although the initial conditions of the neutrino particles were also generated on a grid, soon after the simulation starts, the large neutrino thermal velocities make them to cross the simulation box several times, forcing neutrinos to effectively distribute randomly over the simulation box with a value of their power spectrum equal to $\bar{n}^{-1}$ on all scales. Therefore, when computing the neutrino power spectrum we correct for the neutrino shot-noise by using eq. A.1. The halo distribution is expected to show up as a Poisson process over the underlying matter density field. Thus, we have corrected the halo power spectrum to account for its shot-noise.

When there is more than one fluid, for instance CDM and neutrinos, the total matter power spectrum is related to the individual ones and to their cross-power spectrum by

$$
P_{\mathrm{m}}(\vec{k})=\left(\frac{\Omega_{\mathrm{cdm}}}{\Omega_{\mathrm{m}}}\right)^{2} P_{\mathrm{cdm}}(\vec{k})+\left(\frac{\Omega_{\nu}}{\Omega_{\mathrm{m}}}\right)^{2} P_{\nu}(\vec{k})+\left(\frac{2 \Omega_{\mathrm{cdm}} \Omega_{\nu}}{\Omega_{\mathrm{m}}^{2}}\right) P_{\mathrm{cdm}-\nu}(\vec{k})
$$

where $\Omega_{\mathrm{m}}=\Omega_{\mathrm{cdm}}+\Omega_{\nu}$. As we have seen, the CDM power spectrum does not suffer from shot-noise, and moreover, since the positions of the neutrino and CDM particles do not overlap, the CDMneutrinos cross-power spectrum is not affected by shot-noise [71]. Therefore, the shot-noise of the total matter power spectrum is given by $\left(\frac{\Omega_{\nu}}{\Omega_{\mathrm{m}}}\right)^{2} \bar{n}_{\nu}^{-1}$. We note that this quantity is very small, and 
thus, the correction is, on practice, negligible. Therefore, for simplicity, we decide to ignore the neutrino shot-noise contribution to the total matter power spectrum.

The halo-matter and halo-cold dark matter cross-power spectrums are affected by shot-noise because the halo positions are a subsample of the positions of the CDM particles ${ }^{13}$. The shot-noise level of the cross-power spectrum is given in this case by shot-noise of the matter distribution (see [71] for details), which, as we have seen, is negligible and thus, we do not correct for it.

Finally, since the simulations we have used to compute the bias in Fourier space contains 8 independent realizations, we compute the errors on the measurements of both the power spectrum and the bias just by calculating the standard deviation around the mean value from the whole set.

\section{B Impact of the halo identification criteria on the halo-matter bias}

Here we investigate whether our results, in terms of the bias between the spatial distribution of dark matter haloes and that of the underlying matter, are affected by our simplified assumption of running the FoF and SUBFIND algorithms just on top of the CDM distribution. In order to test the robustness of our results, we run the SUBFIND algorithm over the total matter distribution, i.e. on top of the $\mathrm{CDM}$ plus the neutrino distribution, of the simulations $\mathrm{H} 0, \mathrm{H} 3$ and $\mathrm{H} 6$ to identify the SO haloes. We then compute the bias, $b_{\mathrm{hm}}$ and $b_{\mathrm{hh}}$, for each of the 8 independent realizations for each cosmological model at redshifts $z=0, z=0.5$ and $z=1$ using haloes with masses, $M_{200}$, above $2 \times 10^{13} h^{-1} \mathrm{M}_{\odot}$ and $4 \times 10^{13} h^{-1} \mathrm{M}_{\odot}$. Finally, for each cosmological model, we calculate the mean bias from the results of the 8 independent realizations.

In Fig. 14 we show the ratio of the bias computed by using the SO haloes found on top of the CDM distribution alone to the bias computed by using SO haloes identified on top of the total matter distribution. The left column shows the results for haloes with masses above $2 \times 10^{13} h^{-1} \mathrm{M}_{\odot}$ whereas the right column displays the results for haloes with masses larger than $4 \times 10^{13} h^{-1} \mathrm{M}_{\odot}$. The upper panels show the ratio using the bias computed as $b_{\mathrm{hm}}(k)$ while the bottom panels are for bias computed as $b_{\mathrm{hh}}(k)$. We find that by selecting the dark matter haloes on top of the total matter distribution, our results, in terms of the bias, are basically unchanged. The difference between computing the bias with dark matter haloes selected from the CDM distribution alone and computing the bias with haloes identified from the total matter distribution is less than $\sim 0.5 \%$ for the values of $k$ considered in this work. We conclude that our results are not affected by our simplified assumption of using dark matter haloes as identified from the CDM distribution alone.

\section{References}

[1] C. L. Cowan, Jr., F. Reines, F. B. Harrison, H. W. Kruse, and A. D. McGuire, Detection of the Free Neutrino: A Confirmation, Science 124 (July, 1956) 103-104.

[2] B. T. Cleveland, T. Daily, R. Davis, Jr., J. R. Distel, K. Lande, C. K. Lee, P. S. Wildenhain, and J. Ullman, Measurement of the Solar Electron Neutrino Flux with the Homestake Chlorine Detector, Astrophysical Journal 496 (Mar., 1998) 505.

[3] G. L. Fogli, E. Lisi, A. Marrone, D. Montanino, A. Palazzo, and A. M. Rotunno, Global analysis of neutrino masses, mixings, and phases: Entering the era of leptonic CP violation searches, Physical Review D 86 (July, 2012) 013012, [arXiv: 1205.5254].

[4] D. V. Forero, M. Tórtola, and J. W. F. Valle, Global status of neutrino oscillation parameters after Neutrino-2012, Physical Review D 86 (Oct., 2012) 073012, [arXiv:1205.4018].

[5] J. Lesgourgues and S. Pastor, Massive neutrinos and cosmology, Physics Reports 429 (July, 2006) 307-379, [astro-ph/].

[6] S. Hannestad, Neutrino masses and the number of neutrino species from WMAP and 2dFGRS, Journal of Cosmology and Astroparticle Physics 5 (May, 2003) 4, [astro-ph/].

\footnotetext{
${ }^{13}$ This is because our halo finder assigns the halo center to the position of particle in which the gravitational potential is minimum.
} 

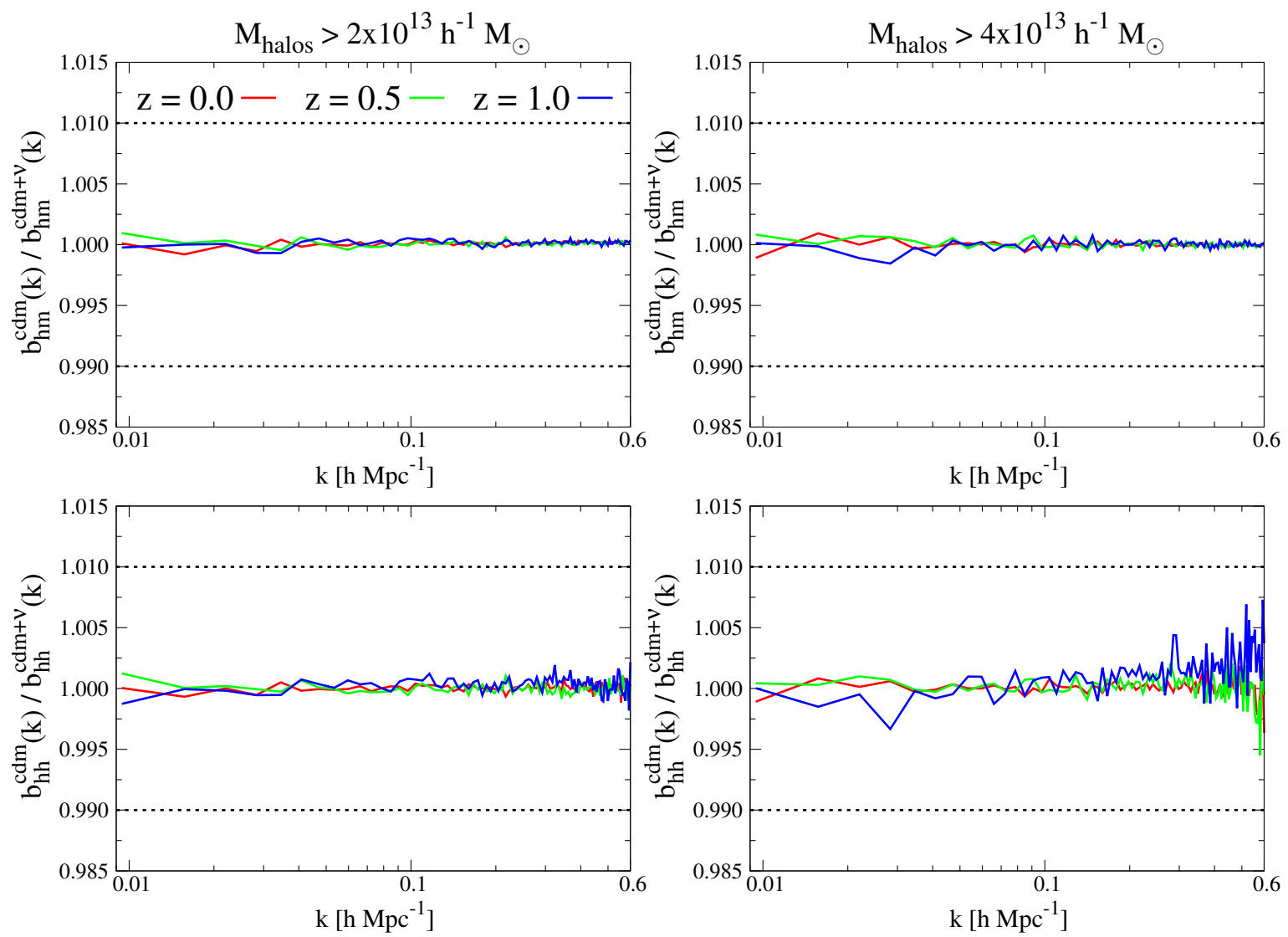

Figure 14. Robustness of our bias results with respect to the halo identification procedure. We compute the bias, $b_{\mathrm{hm}}(k)$ and $b_{\mathrm{hh}}(k)$ by selecting the SO haloes over the total matter distribution. In the panels we plot the ratio of the bias calculated using SO haloes identified over the CDM distribution alone over the bias computed by selecting SO haloes on top of the total matter distribution. The left column shows the results for the haloes with masses, $M_{200}$, above $2 \times 10^{13} h^{-1} \mathrm{M}_{\odot}$ whereas the right column is for haloes with masses above $4 \times 10^{13} h^{-1} \mathrm{M}_{\odot}$. The upper panels show the results for the bias computed as $b_{\mathrm{hm}}(k)$ whereas the bottom panels are for the bias computed as $b_{\mathrm{hh}}(k)$. Results are displayed at $z=0$ (red), $z=0.5$ (green) and $z=1$ (blue). The horizontal dotted lines represent deviations of $1 \%$.

[7] B. A. Reid, L. Verde, R. Jimenez, and O. Mena, Robust neutrino constraints by combining low redshift observations with the CMB, Journal of Cosmology and Astroparticle Physics 1 (Jan., 2010) 3, [arXiv:0910.0008].

[8] S. A. Thomas, F. B. Abdalla, and O. Lahav, Upper Bound of $0.28 \mathrm{eV}$ on Neutrino Masses from the Largest Photometric Redshift Survey, Physical Review Letters 105 (July, 2010) 031301, [arXiv:0911.5291].

[9] M. E. C. Swanson, W. J. Percival, and O. Lahav, Neutrino masses from clustering of red and blue galaxies: a test of astrophysical uncertainties, Montly Notices of the Royal Astronomical Society 409 (Dec., 2010) 1100-1112, [arXiv:1006.2825].

[10] S. Saito, M. Takada, and A. Taruya, Neutrino mass constraint from the Sloan Digital Sky Survey power spectrum of luminous red galaxies and perturbation theory, Physical Review D $\mathbf{8 3}$ (Feb., 2011) 043529, [arXiv: 1006.4845].

[11] R. de Putter, O. Mena, E. Giusarma, S. Ho, A. Cuesta, H.-J. Seo, A. J. Ross, M. White, D. Bizyaev, H. Brewington, D. Kirkby, E. Malanushenko, V. Malanushenko, D. Oravetz, K. Pan, W. J. Percival, N. P. Ross, D. P. Schneider, A. Shelden, A. Simmons, and S. Snedden, New Neutrino Mass Bounds 
from SDSS-III Data Release 8 Photometric Luminous Galaxies, Astrophysical Journal 761 (Dec., 2012) 12, [arXiv:1201.1909].

[12] J.-Q. Xia, B. R. Granett, M. Viel, S. Bird, L. Guzzo, M. G. Haehnelt, J. Coupon, H. J. McCracken, and Y. Mellier, Constraints on massive neutrinos from the CFHTLS angular power spectrum, Journal of Cosmology and Astroparticle Physics 6 (June, 2012) 10, [arXiv:1203.5105].

[13] S. Riemer-Sørensen, C. Blake, D. Parkinson, T. M. Davis, S. Brough, M. Colless, C. Contreras, W. Couch, S. Croom, D. Croton, M. J. Drinkwater, K. Forster, D. Gilbank, M. Gladders, K. Glazebrook, B. Jelliffe, R. J. Jurek, I.-h. Li, B. Madore, D. C. Martin, K. Pimbblet, G. B. Poole, M. Pracy, R. Sharp, E. Wisnioski, D. Woods, T. K. Wyder, and H. K. C. Yee, WiggleZ Dark Energy Survey: Cosmological neutrino mass constraint from blue high-redshift galaxies, Physical Review D $\mathbf{8 5}$ (Apr., 2012) 081101, [arXiv:1112.4940].

[14] G.-B. Zhao, S. Saito, W. J. Percival, A. J. Ross, F. Montesano, M. Viel, D. P. Schneider, D. J. Ernst, M. Manera, J. Miralda-Escude, N. P. Ross, L. Samushia, A. G. Sanchez, M. E. C. Swanson, D. Thomas, R. Tojeiro, C. Yeche, and D. G. York, The clustering of galaxies in the SDSS-III Baryon Oscillation Spectroscopic Survey: weighing the neutrino mass using the galaxy power spectrum of the CMASS sample, ArXiv e-prints (Nov., 2012) [arXiv:1211.3741].

[15] S. More, F. C. van den Bosch, M. Cacciato, A. More, H. Mo, and X. Yang, Cosmological constraints from a combination of galaxy clustering and lensing - II. Fisher matrix analysis, Montly Notices of the Royal Astronomical Society 430 (Apr., 2013) 747-766, [arXiv:1207.0004].

[16] S. Riemer-Sørensen, D. Parkinson, and T. M. Davis, Combining Planck with Large Scale Structure gives strong neutrino mass constraint, ArXiv e-prints (June, 2013) [arXiv:1306.4153].

[17] M. Costanzi Alunno Cerbolini, B. Sartoris, J.-Q. Xia, A. Biviano, S. Borgani, and M. Viel, Constraining neutrino properties with a Euclid-like galaxy cluster survey, Journal of Cosmology and Astroparticle Physics 6 (June, 2013) 20, [arXiv:1303.4550].

[18] T. Basse, O. Eggers Bjaelde, J. Hamann, S. Hannestad, and Y. Y. Y. Wong, Dark energy and neutrino constraints from a future EUCLID-like survey, ArXiv e-prints (Apr., 2013) [arXiv:1304.2321].

[19] C. Carbone, L. Verde, Y. Wang, and A. Cimatti, Neutrino constraints from future nearly all-sky spectroscopic galaxy surveys, Journal of Cosmology and Astroparticle Physics 3 (Mar., 2011) 30, [arXiv: 1012.2868].

[20] Planck Collaboration, P. A. R. Ade, N. Aghanim, C. Armitage-Caplan, M. Arnaud, M. Ashdown, F. Atrio-Barandela, J. Aumont, C. Baccigalupi, A. J. Banday, and et al., Planck 2013 results. XVI. Cosmological parameters, ArXiv e-prints (Mar., 2013) [arXiv:1303.5076].

[21] J. Lesgourgues, G. Mangano, G. Miele, and Pastor, Neutrino cosmology. Apr., 2013.

[22] S. Singh and C.-P. Ma, Neutrino clustering in cold dark matter halos: Implications for ultrahigh energy cosmic rays, Physical Review D 67 (Jan., 2003) 023506, [astro-ph/].

[23] A. Ringwald and Y. Y. Y. Wong, Gravitational clustering of relic neutrinos and implications for their detection, Journal of Cosmology and Astroparticle Physics 12 (Dec., 2004) 5, [hep-ph/04].

[24] J. Brandbyge, S. Hannestad, T. Haugbølle, and Y. Y. Y. Wong, Neutrinos in non-linear structure formation - the effect on halo properties, Journal of Cosmology and Astroparticle Physics 9 (Sept., 2010) 14, [arXiv: 1004.4105].

[25] F. Villaescusa-Navarro, J. Miralda-Escudé, C. Peña-Garay, and V. Quilis, Neutrino halos in clusters of galaxies and their weak lensing signature, Journal of Cosmology and Astroparticle Physics 6 (June, 2011) 27, [arXiv:1104.4770].

[26] F. Villaescusa-Navarro, S. Bird, C. Peña-Garay, and M. Viel, Non-linear evolution of the cosmic neutrino background, Journal of Cosmology and Astroparticle Physics 3 (Mar., 2013) 19, [arXiv: 1212.4855].

[27] J. Brandbyge, S. Hannestad, T. Haugbølle, and B. Thomsen, The effect of thermal neutrino motion on the non-linear cosmological matter power spectrum, Journal of Cosmology and Astroparticle Physics $\mathbf{8}$ (Aug., 2008) 20, [arXiv:0802.3700].

[28] S. Saito, M. Takada, and A. Taruya, Impact of Massive Neutrinos on the Nonlinear Matter Power 
Spectrum, Physical Review Letters 100 (May, 2008) 191301, [arXiv:0801.0607].

[29] J. Brandbyge and S. Hannestad, Grid based linear neutrino perturbations in cosmological N-body simulations, Journal of Cosmology and Astroparticle Physics 5 (May, 2009) 2, [arXiv:0812.3149].

[30] J. Brandbyge and S. Hannestad, Resolving cosmic neutrino structure: a hybrid neutrino N-body scheme, Journal of Cosmology and Astroparticle Physics 1 (Jan., 2010) 21, [arXiv:0908.1969].

[31] S. Saito, M. Takada, and A. Taruya, Nonlinear power spectrum in the presence of massive neutrinos: Perturbation theory approach, galaxy bias, and parameter forecasts, Physical Review D 80 (Oct., 2009) 083528, [arXiv:0907.2922].

[32] M. Viel, M. G. Haehnelt, and V. Springel, The effect of neutrinos on the matter distribution as probed by the intergalactic medium, Journal of Cosmology and Astroparticle Physics 6 (June, 2010) 15, [arXiv: 1003.2422].

[33] S. Agarwal and H. A. Feldman, The effect of massive neutrinos on the matter power spectrum, Montly Notices of the Royal Astronomical Society 410 (Jan., 2011) 1647-1654, [arXiv:1006.0689].

[34] S. Bird, M. Viel, and M. G. Haehnelt, Massive neutrinos and the non-linear matter power spectrum, Montly Notices of the Royal Astronomical Society 420 (Mar., 2012) 2551-2561, [arXiv:1109.4416].

[35] C. Wagner, L. Verde, and R. Jimenez, Effects of the Neutrino Mass Splitting on the Nonlinear Matter Power Spectrum, Astrophysical Journal Letters 752 (June, 2012) L31, [arXiv: 1203.5342].

[36] F. Villaescusa-Navarro, M. Vogelsberger, M. Viel, and A. Loeb, Neutrino Signatures on the High Transmission Regions of the Lyman-alpha Forest, ArXiv e-prints (June, 2011) [arXiv:1106.2543].

[37] F. Marulli, C. Carbone, M. Viel, L. Moscardini, and A. Cimatti, Effects of massive neutrinos on the large-scale structure of the Universe, Montly Notices of the Royal Astronomical Society 418 (Nov., 2011) 346-356, [arXiv:1103.0278].

[38] M. P. van Daalen, J. Schaye, I. G. McCarthy, C. M. Booth, and C. Dalla Vecchia, The impact of baryonic processes on the two-point correlation functions of galaxies, subhaloes and matter, ArXiv e-prints (Oct., 2013) [arXiv:1310.7571].

[39] E. Castorina and et al., Neutrino mass scale-dependence bias, Journal of Cosmology and Astroparticle Physics (Oct., 2013) X, [arXiv:1310.XXXX].

[40] M. Costanzi and et al., The halo mass function in a universe with massive neutrinos: application to galaxy clusters, Journal of Cosmology and Astroparticle Physics (Oct., 2013) X, [arXiv:1310.XXXX].

[41] M. Crocce, P. Fosalba, F. J. Castander, and E. Gaztañaga, Simulating the Universe with MICE: the abundance of massive clusters, Montly Notices of the Royal Astronomical Society 403 (Apr., 2010) 1353-1367, [arXiv:0907.0019].

[42] J. Tinker, A. V. Kravtsov, A. Klypin, K. Abazajian, M. Warren, G. Yepes, S. Gottlöber, and D. E. Holz, Toward a Halo Mass Function for Precision Cosmology: The Limits of Universality, Astrophysical Journal 688 (Dec., 2008) 709-728, [arXiv:0803.2706].

[43] Z. Hou, C. L. Reichardt, K. T. Story, B. Follin, R. Keisler, K. A. Aird, B. A. Benson, L. E. Bleem, J. E. Carlstrom, C. L. Chang, H. Cho, T. M. Crawford, A. T. Crites, T. de Haan, R. de Putter, M. A. Dobbs, and S. Dodelson, Constraints on Cosmology from the Cosmic Microwave Background Power Spectrum of the 2500-square degree SPT-SZ Survey, ArXiv e-prints (Dec., 2012) [arXiv:1212.6267].

[44] V. Springel, The cosmological simulation code GADGET-2, Montly Notices of the Royal Astronomical Society 364 (Dec., 2005) 1105-1134, [astro-ph/].

[45] A. Lewis, A. Challinor, and A. Lasenby, Efficient Computation of Cosmic Microwave Background Anisotropies in Closed Friedmann-Robertson-Walker Models, Astrophysical Journal 538 (Aug., 2000) 473-476, [astro-ph/].

[46] M. Davis, G. Efstathiou, C. S. Frenk, and S. D. M. White, The evolution of large-scale structure in a universe dominated by cold dark matter, Astrophysical Journal 292 (May, 1985) 371-394.

[47] V. Springel, S. D. M. White, G. Tormen, and G. Kauffmann, Populating a cluster of galaxies - I. Results at [formmu2] $z=0$, Montly Notices of the Royal Astronomical Society 328 (Dec., 2001) 726-750, [astro-ph/]. 
[48] A. Dekel and O. Lahav, Stochastic Nonlinear Galaxy Biasing, Astrophysical Journal 520 (July, 1999) 24-34, [astro-ph/].

[49] N. Hamaus, U. Seljak, V. Desjacques, R. E. Smith, and T. Baldauf, Minimizing the stochasticity of halos in large-scale structure surveys, Physical Review D 82 (Aug., 2010) 043515, [arXiv:1004.5377].

[50] T. Baldauf, U. Seljak, R. E. Smith, N. Hamaus, and V. Desjacques, Halo Stochasticity from Exclusion and non-linear Clustering, ArXiv e-prints (May, 2013) [arXiv:1305.2917].

[51] R. E. Smith, R. Scoccimarro, and R. K. Sheth, Scale dependence of halo and galaxy bias: Effects in real space, Physical Review D $\mathbf{7 5}$ (Mar., 2007) 063512, [astro-ph/].

[52] T. Baldauf, R. E. Smith, U. Seljak, and R. Mandelbaum, Algorithm for the direct reconstruction of the dark matter correlation function from weak lensing and galaxy clustering, Physical Review D $\mathbf{8 1}$ (Mar., 2010) 063531, [arXiv:0911.4973].

[53] Y. P. Jing, Correcting for the Alias Effect When Measuring the Power Spectrum Using a Fast Fourier Transform, Astrophysical Journal 620 (Feb., 2005) 559-563, [astro-ph/].

[54] F. Montesano, A. G. Sánchez, and S. Phleps, A new model for the full shape of the large-scale power spectrum, Montly Notices of the Royal Astronomical Society 408 (Nov., 2010) 2397-2412, [arXiv: 1007.0755].

[55] J. L. Tinker, B. E. Robertson, A. V. Kravtsov, A. Klypin, M. S. Warren, G. Yepes, and S. Gottlöber, The Large-scale Bias of Dark Matter Halos: Numerical Calibration and Model Tests, Astrophysical Journal 724 (Dec., 2010) 878-886, [arXiv:1001.3162].

[56] K. Ichiki and M. Takada, Impact of massive neutrinos on the abundance of massive clusters, Physical Review D 85 (Mar., 2012) 063521, [arXiv:1108.4688].

[57] S. D. Landy and A. S. Szalay, Bias and variance of angular correlation functions, Astrophysical Journal 412 (July, 1993) 64-71.

[58] I. Szapudi and A. S. Szalay, A New Class of Estimators for the N-point Correlations, ArXiv Astrophysics e-prints (Apr., 1997) [astro-ph/].

[59] C.-P. Ma and J. N. Fry, Deriving the Nonlinear Cosmological Power Spectrum and Bispectrum from Analytic Dark Matter Halo Profiles and Mass Functions, Astrophysical Journal 543 (Nov., 2000) 503-513, [astro-ph/].

[60] J. A. Peacock and R. E. Smith, Halo occupation numbers and galaxy bias, Montly Notices of the Royal Astronomical Society 318 (Nov., 2000) 1144-1156, [astro-ph/].

[61] U. Seljak, Analytic model for galaxy and dark matter clustering, Montly Notices of the Royal Astronomical Society 318 (Oct., 2000) 203-213, [astro-ph/].

[62] R. Scoccimarro, R. K. Sheth, L. Hui, and B. Jain, How Many Galaxies Fit in a Halo? Constraints on Galaxy Formation Efficiency from Spatial Clustering, Astrophysical Journal 546 (Jan., 2001) 20-34, [astro-ph/].

[63] A. A. Berlind and D. H. Weinberg, The Halo Occupation Distribution: Toward an Empirical Determination of the Relation between Galaxies and Mass, Astrophysical Journal 575 (Aug., 2002) 587-616, [astro-ph/].

[64] A. V. Kravtsov, A. A. Berlind, R. H. Wechsler, A. A. Klypin, S. Gottlöber, B. Allgood, and J. R. Primack, The Dark Side of the Halo Occupation Distribution, Astrophysical Journal 609 (July, 2004) 35-49, [astro-ph/].

[65] Z. Zheng, A. A. Berlind, D. H. Weinberg, A. J. Benson, C. M. Baugh, S. Cole, R. Davé, C. S. Frenk, N. Katz, and C. G. Lacey, Theoretical Models of the Halo Occupation Distribution: Separating Central and Satellite Galaxies, Astrophysical Journal 633 (Nov., 2005) 791-809, [astro-ph/].

[66] I. Zehavi, Z. Zheng, D. H. Weinberg, M. R. Blanton, N. A. Bahcall, A. A. Berlind, J. Brinkmann, J. A. Frieman, J. E. Gunn, R. H. Lupton, R. C. Nichol, W. J. Percival, D. P. Schneider, R. A. Skibba, M. A. Strauss, M. Tegmark, and D. G. York, Galaxy Clustering in the Completed SDSS Redshift Survey: The Dependence on Color and Luminosity, Astrophysical Journal 736 (July, 2011) 59, [arXiv:1005.2413].

[67] M. R. Blanton, D. W. Hogg, N. A. Bahcall, J. Brinkmann, M. Britton, A. J. Connolly, I. Csabai, 
M. Fukugita, J. Loveday, A. Meiksin, J. A. Munn, R. C. Nichol, S. Okamura, T. Quinn, D. P. Schneider, K. Shimasaku, M. A. Strauss, M. Tegmark, M. S. Vogeley, and D. H. Weinberg, The Galaxy Luminosity Function and Luminosity Density at Redshift $z=0.1$, Astrophysical Journal 592 (Aug., 2003) 819-838, [astro-ph/].

[68] I. Zehavi, Z. Zheng, D. H. Weinberg, J. A. Frieman, A. A. Berlind, M. R. Blanton, R. Scoccimarro, R. K. Sheth, M. A. Strauss, I. Kayo, Y. Suto, M. Fukugita, O. Nakamura, N. A. Bahcall, J. Brinkmann, J. E. Gunn, G. S. Hennessy, Ž. Ivezić, G. R. Knapp, J. Loveday, A. Meiksin, D. J. Schlegel, D. P. Schneider, I. Szapudi, M. Tegmark, M. S. Vogeley, D. G. York, and SDSS Collaboration, The Luminosity and Color Dependence of the Galaxy Correlation Function, Astrophysical Journal 630 (Sept., 2005) 1-27, [astro-ph/].

[69] F. C. van den Bosch, S. More, M. Cacciato, H. Mo, and X. Yang, Cosmological constraints from a combination of galaxy clustering and lensing - I. Theoretical framework, Montly Notices of the Royal Astronomical Society 430 (Apr., 2013) 725-746, [arXiv: 1206.6890].

[70] M. Cacciato, F. C. van den Bosch, S. More, H. Mo, and X. Yang, Cosmological constraints from a combination of galaxy clustering and lensing - III. Application to SDSS data, Montly Notices of the Royal Astronomical Society 430 (Apr., 2013) 767-786, [arXiv:1207.0503].

[71] R. E. Smith, Covariance of cross-correlations: towards efficient measures for large-scale structure, Montly Notices of the Royal Astronomical Society 400 (Dec., 2009) 851-865, [arXiv:0810.1960]. 